\author{
Italique \\ Italique \\ Poésie italienne de la Renaissance
}

XXI | 2018

Poesia e Religione

\title{
Osservazioni e riscontri sulle antologie di lirica spirituale (1550-1616)
}

\section{Pietro Giulio Riga}

\section{(2) OpenEdition}

1 Journals

\section{Edizione digitale}

URL: http://journals.openedition.org/italique/621

DOI: 10.4000/italique.621

ISSN: 1663-4438

\section{Editore}

Librairie Droz

\section{Edizione cartacea}

Data di pubblicazione: 1 novembre 2018

Paginazione: $59-98$

ISBN: 978-2-600-05924-4

ISSN: 1423-3983

Notizia bibliografica digitale

Pietro Giulio Riga, «Osservazioni e riscontri sulle antologie di lirica spirituale (1550-1616)», Italique

[Online], XXI | 2018, online dal 01 novembre 2020, consultato il 25 janvier 2021. URL: http://

journals.openedition.org/italique/621 ; DOI: https://doi.org/10.4000/italique.621

(c) Tous droits réservés 
Pietro Giulio Riga

O S S E R V A Z I O N I E R I S C O N T R I

S ULLE ANTOLOGIE D I

L I R I C A S P I R I T U A L E (1 555 0-1 61 l $)$ 



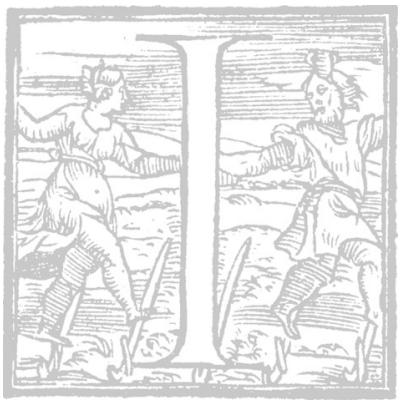

I fondamentale ruolo svolto nel Cinquecento dalle antologie nelle dinamiche di trasmissione di autori e testi poetici è un dato critico ormai acquisito, e gli studiosi che si sono concentrati con sempre maggiore impegno intorno a questo fenomeno hanno messo a fuoco alcune fondamentali prerogative del genere lirico, restituendogli una fisionomia più consona alle sue reali

funzioni estetiche, sociali e linguistiche, come pure al suo retroterra editoriale e commerciale. 'Dopo la prima fondativa serie antologica inaugurata da Giolito nel I545, nitida testimonianza di come si fosse sviluppata la nuova comunicazione poetica in volgare, nelle forme di un petrarchismo agile e flessibile, educato alla scuola di Bembo, ${ }^{2}$ seguirono varie sillogi collettive che differenziarono il loro profilo, caratteriz?andosi tanto per l'ordinamento dei testi, suddivisi per generi o metri, quanto per $i$ registri tematici, valorizzati allo scopo di promuovere una diramazione peculiare del discorso lirico o anche una tradizione alternativa a quella dominante, assolvendo cosi le richieste di una fascia di lettori ampia e trasversale. Nelle nuove miscellanee che irruppero nel panorama librario di secondo Cinquecento le sollecitazioni culturali e letterarie coabitarono strettamente con le ragioni di mercato, in virtù della crescente disponibilità dell'editoria alla diffusione della poesia volgare presso un pubblico qualificato e dalle proporzioni sempre più estese, che poteva cosi agevolmente fruire di prontuari e summae realizzati secondo prospettive di metodo e criteri di selezione specifici. ${ }^{3}$

Nello stesso giro di anni che vide l'uscita dei primi quattro libri delle Rime diverse di molti eccellentissimi autori (I545-I552), entro un contesto convulso e in rapida evoluzione, fu pubblicato anche il primo volume collettivo di poesia spirituale, inangurando una declinazione della forma antologia che, tranne alcuni casi sporadici, non è stata ancora scandagliata in estensione e profondita. ${ }^{4}$ Lo studio che qui si propone mira a colmare, almeno in parte, questa lacuna ricostruendo liter compiuto dalle antologie di lirica spirituale nel corso della seconda metà del XVI secolo, spingendosi fino ai primi decenni del Seicento, periodo nel quale si registra la comparsa di proposte antologiche che registrarono alcuni decisivi cambiamenti in termini di struttura $e$ canone autoriale. Si ritiene utile, pertanto, offrire una panoramica di 
questo sottogenere complementare alla tipologia del libro di «rime di diversì, mettendone in luce l'impianto materiale, le articolazioni strutturali e tematiche. Ove possibile, s'intendono vagliare, sia pure a grandi linee, la storia redazionale, $i$ tempi di allestimento, il contesto di appartenenza, la scansione dei testi, l'organizzazione interna della materia, l'impiego di taluni argomenti dottrinali, considerando che lo status, laico o ecclesiastico, dei curatori influisce necessariamente sulle funzioni culturali, nonché sull'impianto e sulle modalità di fruizione del prodotto antologico. Benché, a prima vista, sembrino dei raggruppamenti indistinti e caotici di testi, derivati da combinazione quasi fortuite, le collettanee di lirica spirituale sono spesso il frutto di una delicata sinergia di fattori che coinvolge la destinazione editoriale, lo sfondo religioso che ne contrassegna la genesi e la ricezione, infine il grado di coscienza e ambizione letterarie. Sarà necessario, poi, considerare l'autonomia progettuale dell'antologia di genere spirituale, che spesso viene allestita secondo convincimenti critico-letterari più espliciti rispetto alle miscellanee «generaliste», con l'intento dichiarato di legittimare la rielaborazione, in chiave sacra, del codice poetico, e in taluni casi tentare di storicizzare un filone lirico allora emergente quale era quello di argomento religioso.

La ricerca si concentrerà su un arco cronologico compreso tra la metà del Cinquecento, che vede l'uscita della prima vera antologia «settoriale» in tre libri (I550-I552), e gli inizi del Seicento, dove si collocano le importanti iniziative di Pietro Petracci - curatore di due antologie, Le Muse sacre (I608) e La celeste lira (I6I2), che riuniscono i piu noti poeti attivi a cavaliere tra $i$ due secoli - e l'antologia di stampo enciclopedico apprestata dal frate domenicano Maurizio Di Gregorio intorno al tema del rosario (I6I4). Grazie alla serie di antologie collocate lungo la parabola evolutiva che va dalla metà del Cinquecento ai primi decenni del secolo successivo la lirica spirituale poteva vantare un canone di riferimento (di autori antichi e moderni) integrato e, per larga parte, sovrapponibile a quello della coeva lirica profana (molti degli autori antologizzati sono letterati di professione, attivi anche sul versante «laico»), ma pur sempre promosso nel quadro di un'offerta culturale ed editoriale che, animata talvolta da ragioni agonistiche, intendeva differenziarsi dichiaratamente da quella di contenuto amoroso o genericamente laicizzante. 
Per avviare un tentativo di ricostruzione organica della fisionomia delle antologie di rime di lirica spirituale e per coglierne le articolazioni nello scenario poetico della seconda metà del Cinquecento occorre iniziare il discorso dall'antologia che inaugurò un nuovo sottogenere tematico all'interno del vasto terreno delle miscellanee liriche, i primi due libri delle Rime spirituali, parte non più stampate, parte novamente da diversi autori raccolte, editi a Venezia presso la libreria veneziana «Al Segno della Speranza» nel I550, seguiti da un Libro terzo che vide la luce due anni più tardi. Per quanto riguarda l'assetto e la composizione materiale della raccolta, su cui si è concentrata soltanto di recente l'attenzione della critica,' basti dire che essa rappresenta il primo tentativo di organizzazione selettiva della produzione lirica di carattere religioso, che comprende un arco piuttosto esteso della tradizione volgare, dai versi di Vittoria Colonna e del Petrarca spirituale di Malipiero, ai recuperi di sapore «archeologico» dei testi di Cariteo e di Girolamo Benivieni inseriti nel secondo volume e delle laudi di Leonardo Giustinian e di altri rimatori presenti nel terzo e ultimo tomo. Uno spoglio che si svolgeva prevalentemente sulle stampe circolanti in quegli anni a Venezia (in particolare, per quanto riguarda il primo libro, sulle antologie giolitine uscite proprio a ridosso del Is5o, con una preferenza accordata al secondo libro del I547) condotto con un'attitudine selettiva complessivamente più meditata e storiograficamente avveduta rispetto all'archetipo delle Rime diverse, che aveva operato una rimozione pressoché assoluta di ogni testimonianza poetica di antica tradizione. L'antologizzatore delle Rime spirituali, invece, non intende soltanto volgere la poesia religiosa verso il «moderno», ma vuole offrire di quell'esperienza un disegno coerente e omogeneo, fissando una gerarchia di autori e di testi cronologicamente e culturalmente più aperta rispetto a quella che le raccolte giolitine stavano proprio allora definendo in maniera onnicomprensiva. Questa raccolta antologica viene dunque prendendo una forma caratteristica, che, al contrario di altre miscellanee, entro le quali venivano riversate, secondo una ratio spesso approssimativa se non, in taluni casi, opportunistica, i materiali testuali allora disponibili ai curatori, ambisce anche a tracciare, in termini ovviamente impliciti, una storia della lirica di materia spirituale, delineando un percorso di lettura razionale, scandito per forme metriche: il primo volume è composto da sonetti, il secondo da vari metri, soprattutto canzoni e madrigali, il terzo da laudi. ${ }^{6}$ Le Rime spirituali risponde- 
vano dunque a una duplice vocazione: da una parte offrivano una panoramica di ciò che la lirica contemporanea di argomento religioso aveva prodotto sino a quella data, dall'altra, con gli innesti del secondo e terzo volume, guardavano a ritroso, recuperando alcuni prodotti della poesia quattrocentesca esenti ancora dall'uniformazione petrarchistica. Il metodo di raccolta dei testi non è, dunque, casuale, ma sembra rispondere a un progetto preciso, che vuole amalgamare voci poetiche di diversa estrazione cronologica e culturale, soprattutto attraverso il recupero della lauda, che rappresenta, lo si sa, l'esordio della poesia volgare religiosa in Italia. ${ }^{7}$ E sulla volontà di recuperare questa forma metrica sembra pesare direttamente la politica editoriale che di li a pochi anni caratterizzerà l'impresa "Al Segno della Speranza», dai cui torchi uscirono nel I5s6 un'edizione di laude iacoponiche e un'antologia laudistica di autori moderni. ${ }^{8}$

Nei tre tomi che compongono la serie, di cui allo stato attuale delle ricerche risulta difficile ricostruire $i$ tempi e $i$ modi di realizzazione, spogli come sono di paratesti e apparati informativi convivono le consuete opportunità editoriali e commerciali che condizionano la fisionomia di ogni antologia lirica, con le ragioni di un progetto culturale che vuole mostrare la pari dignità della poesia spirituale rispetto a quella profana, canonizzandone non solo gli autori di rango (Colonna e Malipiero), ma anche gli interpreti meno blasonati, al fine di attraversare obliquamente il sottogenere. Alcune tra le maggiori esperienze della poesia spirituale in volgare venivano archiviate entro un percorso di lettura che segnalava, a ritroso, come alle prove quattrocentesche fosse seguita una stagione di intenso fermento creativo, nel quale il linguaggio e gli strumenti tecnici della poesia profana erano stati assorbiti e rivolti alla trasmissione di contenuti religiosi, senza però difettare in coerenza retorica e tenuta formale.

Il Libro primo è quello che più di tutti si avvicina, per articolazione, scelta metrica e impianto materiale (un libretto in ottavo di 199 carte folte di autori e testi, realizzato per una compulsazione ricorrente), alle prime uscite della serie giolitina.' L'ampio e composito corpus degli autori è accomunato dalla forma metrica più impiegata in ogni antologia coeva, il sonetto, e accosta sistematicamente noti letterati professionisti con autori «mezani», se non proprio dilettanti, privi di propri libri di poesia, aspetto che testimonia come le loro rime siano state prelevate soprattutto da precedenti offerte antologiche. Come accade in tutte le 
miscellanee dell'epoca, anche le Rime spirituali vincolano le proporzioni quantitative all'esemplarità del valore di ciascun autore; bisogna tuttavia tenere a mente che le scelte furono vincolate anche a questioni contingenti, tanto all'offerta disponibile sul mercato librario quanto a ragioni di amicizia personale o prossimità geo-culturale (in questo caso Venezia e il Veneto), che giocoforza tendono a depotenziare il valore del numero in relazione alla qualità e all'importanza degli autori antologizzati.

L'insieme eterogeneo dei rimatori del Libro primo delinea con nitore il profilo della lirica spirituale di medio Cinquecento, una lirica strettamente intrecciata alle forme del petrarchismo, lungo una linea che poneva ai vertici del canone Vittoria Colonna e Girolamo Malipero, collocati al centro del Libro primo, la prima con $2 I 2$ sonetti (oltre al Primo capitolo del triompho di Christo), ${ }^{10}$ il secondo con $i$ 3I7 sonetti del Petrarca spirituale. " ${ }^{\text {I }}$ L'ordine dei componimenti lascia supporre che $i$ testi della Colonna siano stati prelevati in blocco dall'edizione Valgrisi delle Rime spirituali del I548, mentre i testi di Malipero provengono con ogni probabilità da una stampa veneziana del Petrarca spirituale. Se quindi nella silloge venivano esaltati gli autori che sono tutt'oggi considerati $i$ più rappresentativi del petrarchismo spirituale di prima generazione, agli estremi del volume si disponeva un insieme frastagliato di 50 autori per un totale di I 85 testi (7 dei quali di «incerti autori»). Questa la tavola in ordine decrescente, nella quale si fa seguire ai nomi il numero dei testi con $i$ quali ciascun autore è attestato nella raccolta:

Alessandro Piccolomini (36), Giovanni Del Bene (27), Ludovico Dolce (i 6), Antonio Agostino Torti (I 3), Camillo Besalio (I 3), Parthenio Suavio [Colantonio Carmignano] (I 2), Giovanni Guidiccioni (I I), Ludovico Pascale (9), Antonio Mezzabarba (8), Giovan Battista Giraldi Cinzio (8), Luigi Alamanni (7), Incerti autori (7), Giovan Agostino Caccia (7), Iacopo Sannazaro (5), Pietro Aretino (5), Girolamo Parabosco (5), Laura Terracina (4), Giovan Giacomo Del Pero (4), Tommaso Castellani (3), Amomo (2), Fortunio Spira (2), Bernardino Tomitano (2), Veronica Gambara (2), Claudio Tolomei (2), Francesco Maria Molza (2), Luca Valenziano (2), Anton Giacomo Corso (2), Baldassar Stampa (2), Vincenzo Martelli (2), Antonio Girardi (2), Remigio Fiorentino (2), Bernardo Tasso (I), Pietro Bembo (I), Paolo Crivello (I), Benedetto Varchi (I), Bernardino Daniello (I), Alessandro Campesano (I), 
Pietro Giulio Riga

Ludovico Domenichi (I), Anton Francesco Raineri (I), Bartolomeo Ferrino (I), Astemio Bevilacqua (I), Felice Figliucci (I), Giovanni Giustiniano (I), Petronio Barbato (I), Tullia D’Aragona (I), Francesco Maria Guglia (I), Francesco Maria Molza (I), Lelio Capilupi (I), Bernardino Tomitano (I), Vittoria Colonna (I), Nicolò Amanio (I).

A una valutazione cursoria della tavola dei componimenti si evince che alcune sequenze sono state riprese dalle antologie dei Giolito, in linea con un'attitudine al riuso e all'assemblaggio di materiali di riporto comune a tutte le antologie poetiche. ${ }^{\mathrm{I}}$ Gli II testi di Giovanni Guidiccioni e is di Pietro Aretino derivano dal Libro primo della giolitina, mentre tutti quelli ascritti a Giovanni Del Pero, Antonio Mezzabarba e Lodovico Dolce sono prelevati dal Libro secondo delle Rime di diversi, che registra un aumento delle composizioni di carattere religioso. Per non parlare, poi, di tutti quegli autori attestati con uno o due componimenti, la cui provenienza è nella maggioranza dei casi da ricondurre a un precedente antologico. ${ }^{\mathrm{I}}$ Ovviamente, anche le raccolte d'autore offrono materiali al volume, secondo dinamiche selettive piu motivate da ragioni di gusto; ad esempio, il nucleo di testi di Alessandro Piccolomini si legge nei Cento sonetti, editi da Valgrisi nel I549, gli 8 testi di Giraldi Cinzio sono ricavati dalle Fiamme, pubblicate proprio da Giolito nel I548, mentre i 7 pez:i di Alamanni risalgono quasi certamente a un'edizione veneziana delle Opere toscane.

La capacità di selezionare da precedenti nuclei poetici a stampa diventava per l'anomimo curatore delle Rime spirituali il principale compito intellettuale, secondo un metodo di campionatura che faceva del criterio selettivo, e in secondo luogo di quello quantitativo, un mezzo per attribuire un valore esemplare e normativo agli autori prescelti, nonché per promuovere e in qualche modo realizzare una sistemazione storicocritica di un genere, quello della poesia spirituale, che, quantunque rivendicasse una tradizione autonoma, non si era mai conquistato una propria centralità nel panorama letterario della prima metà del Cinquecento. Si dovrà dunque guardare a questa operazione come a un primo sforzo di definizione storiografica della tradizione lirico-religiosa, fondata soprattutto (con l'eccezione macroscopica di Malipiero) sulla produzione di autori «laici», in un periodo nel quale la separazione tra discorso sacro-morale e discorso amoroso non era ancora stata tracciata in maniera cosi netta come accadrà invece dagli anni Sessanta in avanti. 
Per come si articola, il primo tomo delle Rime spirituali si lega fortemente all'economia culturale e organizzativa dei recenti libri di poesia petrarchista (sia individuali sia collettivi), diventando di quelle proposte una sorta di appendice specialistica che instaura pur sempre un legame elettivo con $i$ Fragmenta petrarcheschi, entro un sistema di filtrazione, citazione e riscrittura del modello.

Converrà a questo punto ragionare sul senso di alcune inclusioni. Se il Petrarca spirituale di Malipero e le liriche di Vittoria Colonna rispondono alla lucida cognizione del ruolo fondativo di queste due figure nel quadro del petrarchismo spirituale, ${ }^{\mathrm{I} 4}$ gli autori che seguono per numero di testi, Piccolomini, Del Bene, Dolce e Torti, delineano un canone atipico ma culturalmente significativo. Alla base della scelta di Torti e Del Bene (quest'ultimo, per di più, compare come primo autore in tutti e tre $i$ volumi della raccolta), i cui testi risultano per scelte metriche e struttura argomentativa parzialmente estranei al modello petrarchesco, si può ipotizzare, da parte del curatore, una motivazione personale e, al contempo, geo-culturale, trattandosi di autori interni al contesto riformistico veronese guidato da Gian Matteo Giberti prima e da Alvise Lippomano poi. ${ }^{15}$ Anche qui, dunque, come in altri volumi collettanei, la silloge è influenzata da una vasta dinamica di fattori, che se da un lato rispondono a precise istanze culturali, dall'altro sembrano nascere da congiunture legate ad esigenze personali o alle vicende di reperimento dei testi. Tuttavia, di là da ragioni contingenti, se è vero, come detto, che in ogni selezione antologica il primato quantitativo puo sottintendere una griglia qualitativa, sorprende che Pietro Bembo, presente soltanto con il sonetto Alto Re, se la mia piu verde et calda, sia cosi sottostimato, forse perché al padre fondatore della lirica volgare non era riconosciuta quella solida ispirazione religiosa che animava autori molto meno noti ma numericamente più rappresentati nella silloge.

Sul fronte contenutistico, nel Libro primo sono lumeggiati conflitti interiori e percorsi di fede interiorizzati, oscillanti tra pentimento e preghiera, in piena sintonia con l'eredità poetica del "Petrarca spirituale», ${ }^{16}$ dove sono privilegiati il tema cristologico e gli episodi neotestamentari. Anche il sostrato dottrinale risulta piuttosto variegato, in linea con il contesto teologico e istituzionale ancora fluido e plurale degli anni Trenta e Quaranta; senza entrare nel merito di un discorso troppo ampio e delicato, che obbligherebbe a unadigressione in merito 
alle esperienze religiose di primo e medio Cinquecento affidate alle scritture poetiche, merita di essere segnalata la presenza di tre sonetti di Guidiccioni in lode di Bernardino Ochino risalenti al I538, agli anni, quindi, dellintensa attività di predicatore che precede l'esilio in Svizzera. ${ }^{17}$

L'impianto complessivo dei tre libri delle Rime spirituali nasce dunque dalla compresenza di varie esigenze, mirate all'appagamento delle richieste, ormai sempre più pressanti, di un pubblico affamato di scritture religiose, verso cui il mondo editoriale si stava rivolgendo con sempre maggiore energia e applicazione. Pur essendo nata nel solco delle sillogi a stampa che la precedono, l'operazione rivendicava una propria specificità sia per la scelta degli autori sia per l'articolazione interna, oltre che, come anticipato, per la ripresa di una forma metrica estranea alla tradizione petrarchista come la lauda, genere che godrà di nuovo slancio tra Cinque e Seicento grazie anche a una fortunata antologia, il Libro primo delle laudi spirituali composte da «diversi eccellenti e divoti autori antichi e moderni》 allestita dal domenicano fiorentino Serafino Razzi. ${ }^{18}$ Si tratta di una silloge che nasce da un'esigenza liturgica, destinata a un pubblico devoto composto prevalentemente da «semplicis, ${ }^{19}$ che concerne la declamazione corale, pubblica, del patrimonio laudistico «in Firenze, dopo il Vespro o la Compieta, a consolazione e trattenimento de' divoti servi di Dio». ${ }^{20}$ In linea con la fase espansiva, per esiti editoriali, della tradizione lirico-musicale, la raccolta assegnava alle laudi, intese genericamente da Razzi come «canzoni spirituali», un margine di autonomia entro il campo della nuova scrittura religiosa in volgare al servizio della formazione religiosa del fedele, diventando a tutti gli effetti il genere di poesia cantata, a una o più voci, piu diffuso nell'Italia post-tridentina. ${ }^{21}$ Dopo le prime uscite a stampa delle laudi iacoponiche, ${ }^{22}$ la silloge valeva come prima esplicita canonizzazione editoriale del genere laudistico e si rivolgeva a un pubblico di religiosi e religiose, riunendo componimenti, con relativa partitura musicale, di 22 autori, prevalentemente quattrocenteschi, la maggioranza dei quali di origine fiorentina e toscana; l'apertura era affidata a Razzi stesso, cui spettava il dominio assoluto dei testi nella raccolta, che mantiene un certo disordine strutturale per via di autori presenti in diverse parti del volume. Questo l'elenco dei nomi: Feo Belcari, Angelo Bettini, Bianco dall'Ancolina, Ilario Buoninsegni, Pierfelice Caiani, Castellano Castellani, Felice da Castelfranco, Gherardo d'Astore, Marco Della 
Casa, Niccolò Fabroni, Leonardo Giustinian, Bonifacio Landini, Battista Malatesta, Iacopo Mariscotti, Lorenzo de' Medici, Simon Pallaio, Francesco Petrarca, Girolamo Savonarola, Lorenzo Tornabuoni, Lucrezia Tornabuoni e Benedetto Varchi.

Da questa rassegna, che esalta la tradizione laudistica toscana, a cominciare dal magistero assunto da Feo Belcari, ${ }^{23}$ conta rilevare la presenza della canzone petrarchesca Vergine bella, a segnalare la straordinaria fortuna di questo testo, sradicato dal corpus poetico originario, nei territori della fruizione devota, ${ }^{24}$ e soprattutto della traduzione varchiana del Miserere, dato, quest'ultimo, che mette in stretta relazione la lauda con un altro genere spirituale fondato sul canto e sulla preghiera come il salmo, oggetto nel corso del Cinquecento di varie rielaborazioni poetiche in volgare. ${ }^{25}$

Il successo culturale e commerciale riscosso dal genere delle antologie sollecitò l'allestimento di progetti analoghi a quello dei tre libri delle Rime spirituali, benché non sempre dotati di simili estensioni e ambizioni culturali. Un'eccezione di rilievo sono, in ragione di una selezione autoriale attentamente meditata, le Rime spirituali di sette poeti illustri, stampate a Napoli nel Is6o per le cure di Scipione Ammirato, firmatario della lettera di dedica a Geronima Colonna. ${ }^{26}$ L'esile silloge richiamava il modello dei Carmina quinque illustrium poetarum italorum, editi per la prima volta nel I548, giovandosi tuttavia degli esempi delle coeve sillogi in volgare, la selezione operata nei ruscelliani Fiori delle rime de' poeti illustri, vero e proprio specimen del petrarchismo lirico, ${ }^{27}$ e soprattutto le Rime di tre de' più illustri poeti dell'età nostra, uscite a Venezia nel I567, che riunivano tre autori presenti anche nella raccolta di Ammirato: Bembo, Guidiccioni e Della Casa. ${ }^{28}$ Sembra dunque chiaro che lo scrittore salentino voglia proporre una soluzione concorrente alle raccolte poetiche "profane» attingendo tuttavia dallo stesso fonte, ossia dalla traiettoria piu alta del petrarchismo rinascimentale. Al metodo selettivo dell'antologia si allude in un passo della dedicatoria, nella quale Ammirato discorre della «scelta» come di una

cosa benché piccola, nondimeno, come sono le gioie, assai cara e preziosa, sì per il soggetto ch'elle contengono e sì per la degnità de i loro autori, tra' quali tenendo luogo assai chiaro et illustre Vittoria Colonna, parvemi eziandio per questo rispetto cosa convenientissima dedicarle a Geronima Colonna come principal Signora dell'istessa famiglia e sua 
nipote, così grandemente immitatrice et emula del suo veramente sovra umano e quasi divino valore. ${ }^{29}$

Occorre dunque dare nota della successione degli «illustri» che Ammirato trasceglie scrupolosamente dal mare magnum della rimeria coeva: Francesco Petrarca (9), Pietro Bembo (9), Giovanni Guidiccioni (II), Francesco Maria Molza (9), Giovanni Della Casa (6), Vittoria Colonna (38), Berardino Rota (39). I campioni assoluti della silloge sono Rota e Colonna, a testimoniare la volontà di trasferire il baricentro geoculturale del volume in area napoletana. ${ }^{30}$ Ma se Rota sigla il volume designando, secondo Ammirato, l'apice del filone sacro-morale della lirica volgare, l'autore chiamato a svolgere la funzione di apripista è Petrarca, cui viene affidato il ruolo di auctoritas anche del sottogenere spirituale. Seguono Bembo e Della Casa, apostoli, secondo Ammirato, di un nuovo itinerario, più impegnativo e impervio, della lirica, marcato da uno stile «ritenuto e grave», che segna una ridefinizione stilisticotematica del grande archetipo trecentesco. ${ }^{3}$

Petrarca, dunque, cui devono guardare tutti i rimatori spirituali come a un esempio insuperato di lingua e stile, segna le prime pagine dell'antologia, nelle quali si segnalano, oltre al sonetto Padre del Ciel $e$ allinevitabile Vergine bella, il Triumphus Temporis $e$ il Triumphus Eternitatis. Poi è la volta di Bembo, che tallona Petrarca collocando l'operazione antologica di Ammirato nell'alveo della tradizione lirica volgare e del sistema petrarchesco; $i$ nove componimenti bembiani (8 sonetti e I ballata), variazioni sul tema della preghiera a Dio (scanditi dalla serie che inizia con Signor) e alla Vergine, sono estratti soprattutto dalla sezione finale del liber di Bembo, che accoglie testi di lode, confessione e pentimento, culminante con la ballata Signor, quella pietà che ti constrinse che sigla il macrotesto bembiano. ${ }^{32}$ Sulla scia della lezione di Petrarca e Bembo, Ammirato innesta gli esiti maggiori del petrarchismo lirico di medio Cinquecento, trascegliendo i versi di ispirazione religiosa. Dopo le sezioni dedicate a Guidiccioni ${ }^{33}$ e Molza, fa il suo l'ingresso Della Casa, la cui poesia, come detto, rappresenta per Ammirato un modello di eccellenza formale e, sulpiano etico-religioso, un perfezionamento dell'esempio dei Rerum vulgarium fragmenta; la sua presenza si riduce, è vero, a soli sei componimenti, ma tra i più significativi della stagione che inaugura la poetica della gravitas, con $i$ due capisaldi rappresentati dal sonetto 
Questa vita mortal e dalla canzone Errai gran tempo. ${ }^{34}$ Infine, si giunge al vertice del canone designato da Ammirato, ai due assi portanti della silloge, Colonna e Rota, che distaccano in termini quantitativi $i$ rimatori che li precedono: mentre la porzione dedicata a Rota è costruita offrendo in prevalenza materiali inediti assemblati da Ammirato (3I testi dei 39 totali), ${ }^{35}$ approfittando del rapporto di amicizia e di stretta collaborazione intellettuale allacciato tra l'autore e il curatore sul piano critico ed editoriale, ${ }^{36} i{ }^{8} 8$ testi della Colonna sono ripresi da alcune edizioni delle Rime della marchesa di Pescara. ${ }^{37}$ Sul piano critico, la selezione offerta da Ammirato appare lucida e culturalmente aggiornata, e rispecchia pienamente il canone che si andava costruendo in quegli anni anche sul fronte laico, dove la moda della gravitas si era imposta con successo, sollecitando uno sconfinamento tematico della lirica nei settori della riflessione morale e del discorso religioso. ${ }^{38}$

Sempre a Napoli, qualche anno più tardi, videro la luce le Rime spirituali di diversi eccellenti poeti toscani, edite nel Is74 per le cure dello scrittore foggiano Giovan Battista Vitale, ${ }^{39}$ autore in proprio di un volume di Rime piacevoli [...] con alcuni centoni di versi del Petrarca, edito a Orvieto nel I598. Su questa figura poco o nulla è possibile ricavare da stampe coeve e repertori eruditi, quindi converrà limitarsi all'analisi del volume, che presenta un quadro delle presenze piuttosto eterogeneo; nella lettera di dedica a Vittoria Sanseverino, datata Napoli, I8 aprile I574, Vitale afferma di voler offrire alla duchessa di Termoli un "picciolo e utilissimo libro delle rime spirituali di diversi», conveniente alla sua statura morale per via della «riputazion degli autori», l'«altezza e varietà del soggetto», la «candidezza e dolcezza delle rime»..$^{40}$ Una selezione, dunque, indirizzata a una colta e influente nobildonna, e dunque concepita per un pubblico colto, di laici intendenti, dove la componente squisitamente letteraria prevale su quella devota e pastorale. Per comprendere quale tipo di selezione ba operato Vitale si riporta la successione degli autori nel volume, disposti secondo un criterio alfabetico:

Anton Giacomo Corso (I), Antonio Mezzabarba (4), Anton Francesco Raineri (I), Bartolomeo Ferrino (3), Bernardino Tomitano (2), Bernardino Daniello (I), Berardino Rota (29), Camillo Besalio (6), Claudio Tolomei (3), Domenico Venier (2), Francesco Petrarca (6), Francesco Maria Molza (9), Francesco Angelo Coccio (I), Francesco Capodilista (I), Iacopo Sannazaro (4), Giacomo Marmitta (I), Giacomo Cenci (I), 
Pietro Giulio Riga

Giovanni Guidiccioni (7), Giovanni Della Casa (5), Giovanni Andrea dell'Anguillara (3), Giovan Battista Susio (I), Giovan Giacomo Benalio (3), Giovan Giacomo del Pero (5), Girolamo Troiano (2), Incerto Autore (2), Lodovico Ariosto (I), Lodovico Domenichi (I), Lodovico Novello (I), Lodovico Dolce (I 2), Michelangelo Buonarroti (2), Paolo Crivello (I), Paolo Belvedere (3) Petronio Barbati (I), Pietro Bembo (9), Sansonetto Sansonetti (I), Tommaso Castellani (2), Veronica Gambara (I), Vittoria Colonna (36).

Anche qui Vitale, seguendo una prassi consolidata, seleziona una parte dei 174 testi dei 37 autori complessivi dalle miscellanee poetiche pubblicate negli anni precedenti. Sulla scelta di un ristretto ma prestigioso gruppo di poeti pesa direttamente il modello antologico delle Rime spirituali di sette poeti illustri, dal quale Vitale estrae $i$ testi per le analoghe partizioni di Petrarca, Bembo, Guidiccioni, Molza, Della Casa, Colonna e Rota, rispettandone spesso anche l'ordinamento. Per rimpinguare la miscellanea Vitale si serve anche di altre antologie cinquecentesche, permettendo cosi che le rime di alcuni poeti non proprio di primo livello continuino a circolare, in virtù della loro massiccia presenza nelle sillogi precedenti: caso più eclatante è quello di Lodovico Dolce (terzo per numero di testi), di cui sono attestati ben dodici sonetti apparsi prima nel secondo libro delle Rime di diversi nel Is 47 e poi nel primo libro delle Rime spirituali del Isso. Stupisce, infatti, che Dolce, privo di una propria raccolta poetica a stampa e legato a una stagione editoriale e letteraria ormai superata, continui a recitare la parte di protagonista, occupando una posizione di assoluto rilievo, in termini quantitativi in una antologia degli anni Settanta. Converrebbe dunque chiedersi se si tratta di inerte o consapevole ripresa di testi dai contenuti, peraltro, potenzialmente pericolosi, intrisi come sono di riferimenti a questioni dottrinali scottanti (giustificazione per sola fede, valore delle opere, grazia divina, illuminazione interiore, beneficio di Cristo), che non ci si aspetterebbe di ritrovare in una antologia di questi anni, dopo che $i$ decreti tridentini si erano pronunciati apertamente sulle maggiori controversie in materia di fede. ${ }^{4 \mathrm{I}}$ La questione, tuttavia, meriterebbe uno studio a parte, che prenda in considerazione la ricezione di testi come questi da parte degli utenti dell'epoca (editori, lettori, censori), da estendere, ovviamente, anche al caso di Vittoria Colonna, eccezionalmente rappresentata, anche in tempi di rigorismo posttridentino, nelle proposte di Ammirato e Vitale. 
Consultando la banca dati Lyra (ex Alirasta) si evince che anche la serie di 6 componimenti di Besalio e i 4 di Mezzabarba sono recuperati con ogni probabilità dalle Rime spirituali del I550, mentre $i_{3}$ testi di Ferrino e is di Del Pero sono verosimilmente un retaggio del secondo libro dell'antologia giolitina del I547. Un altro drappello di rimatori, ciascuno dei quali presente con un basso numero di testi (dell'Anguillara, Benalio, Buonarroti, Tolomei, Venier, Troiano, ecc.), proviene, invece, dalle Rime di diversi nobili poeti toscani raccolte da Dionigi Atanagi e pubblicate in due tomi a Venezia per $i$ tipi di Ludovico Avanzi nel biennio Is65-66. Per i 4 componimenti di Sannazaro, il foggiano invece abbandona volumi antologici e attinge direttamente da un'edizione dei Sonetti et canzoni.

Dalla cursoria analisi che si è qui proposta è emerso che Vitale seleziona gran parte dei testi dalle varie sillogi collettive disseminate sul panorama librario, attribuendo, in linea con la prospettiva meridionalizzante assunta nell'antologia di Ammirato, un ruolo di guida alla poesia di Rota, secondo soltanto alla Colonna, cui seguono gli altri «excellenti», appartenenti per lo più alla generazione dei nati prima del I500 o nei primi anni del secolo XVI (Molza, Sannazaro, Guidiccioni, Della Casa, Bembo, ecc.). Per Vitale non si trattava più, come era stato, almeno in parte, per l'anonimo redattore delle Rime spirituali del I550-52, di allestire un volume che ribadisse la legittimità e la piena autonomia della lirica spirituale nel quadro della moderna poesia in volgare, ma di avviare, secondo un'impostazione critica personale, o comunque rispondente al contesto nel quale fioriva l'operazione editoriale, una canonizzazione di ciò che di meglio aveva offerto la più recente stagione della lirica di argomento religioso. D'altro lato, l'innesto di sei componimenti petrarcheschi assume anche qui la funzione di dichiarare il valore assoluto di quel modello normativo, trasmettendo maggiore organicità a una silloge nella quale la pratica della poesia spirituale deriva principalmente da un'amplificazione discorsiva del dettato petrarchesco e, di conseguenza, viene intesa come una diramazione settoriale del petrarchismo tout court.

Di particolare rilievo risulta anche l'esile manipolo di Rime spirituali di diversi illustrissimi cardinali, di reverendissimi vescovi e d'altre persone ecclesiastiche edite a margine dei Salmi penitentiali di diversi eccellenti autori, che, apparsi per i tipi di Giolito nel I568, ristampati l'anno seguente e riediti con alcune variazioni nel I572, 
offrirono la prima canonizzazione del sottogenere poetico delle riscritture salmiche in volgare, nelle quali si assiste a una rielaborazione ancora piu profonda e innovativa del codice formale e linguistico petrarchesco, riletto alla luce del modello davidico. ${ }^{42}$ La partizione delle rime ospita un corpus quantitativamente smilzo ma culturalmente importante, sia perché riunisce figure di peso nel panorama letterario cinquecentesco, sia perché, per la prima volta, gli autori sono trascelti in quanto prelati e uomini di Chiesa, aspetto che marca l'elemento catechistico e pastorale connaturato alla silloge. La precisazione accolta nel frontespizio del volume è degna di nota e va intesa come una spia del mutamento storico-cultarale in corso, nel quadro di una ridefinizione degli obiettivi e della collocazione sociale della poesia lirica di argomento religioso, praticata e promossa, dopo la svolta di Trento, da una solida milizia di chierici che, mossa da finalità educative e precettistiche, intese dimostrare come la lirica potesse misurarsi con materie religiose e il diletto poetico scaturire anche dalla frequentazione di tematiche sacre. Tale fenomeno derivava anche dalla nuova strategia culturale messa in atto dalla Chiesa di Roma, che esercitò dagli anni Sessanta in avanti un controllo costante nei confronti dell' attività intellettuale, e quindi del medium tipografico, controllo che si farà via via sempre più scrupoloso e pervasivo grazie all'azione svolta dalla censura, in concomitanza con la promulgazione dell'Indice paolino nel $1559 .^{43}$ Al pari di altre forme di scrittura (orazioni, trattati, agiografie, catechismi, breviari e libri di preghiera), anche il genere lirico fu impiegato come strumento di edificazione del fedele e investito di quella cultura della direzione spirituale, innescata dagli Esercizi spirituali di Ignazio di Loyola, che fu un tratto distintivo del cattolicesimo post-conciliare, ${ }^{44}$ collocandosi al crocevia di due tipologie librarie ben distinte sul mercato editoriale, la raccolta di rime di derivazione petrarchista e il libro di argomento devoto indirizzato alla formazione del fedele laico ed ecclesiastico. ${ }^{45}$

Tornando alla silloge, risaltano per chiarezza programmatica le considerazioni del curatore della raccolta, il carmelitano Francesco Turchi, che nella lettera di dedica a Laura Pola allude al progetto di Gabriel Giolito di «arricchire il mondo co' libri cristiani fatti nelle sue vaghissime stampes. ${ }^{46}$ L'intento di Turchi è quello di bilanciare l'elemento devoto di un libro finalizzato principalmente alla meditazione e alla preghiera con istanze piu squisitamente letterarie, sottolineando $i$ benefici che possono ricavare i fedeli dalla lettura di quei versi: 
Onde io prego Vostra Signoria Illustre a volerlo accettare con lieto e grato animo, non risguardando alla quantità ma alla qualità di lui, nel quale (oltre i Salmi penitenziali) vi leggerete anco di vaghi e leggiadri componimenti di diversi Illustrissimi e Reverendissimi Prelati. Ne' quali componimenti si scorge la vana opinione d'alcuni che si danno a credere non potersi scrivere poeticamente bene i concetti pii e cristiani. Là onde e gli uomini e le donne spirituali, con tutte quelle persone modeste che si dilettano di leggere libri di poesia, potranno ora (senza aver nelle mani libri che soglion dare ad alcuni scrupolosi qualche sospetto d'immodestia), leggendo questo libro, insieme dilettare il senso et lodare Iddio. ${ }^{47}$

Il passo ospita, secondo una prassi consolidata, una notazione relativa al divario della lirica spirituale rispetto a quella di argomento mondano, basato sulla sostituzione della materia poetabile, che percorre ora versanti tematici alternativi a quelli della «vulgata» petrarchista, al fine di rimodulare l'esercizio lirico in una direzione propriamente didattica, dimostrando come alcuni dei più rinomati poeti cinquecenteschi siano stati capaci di verseggiare intorno a «concetti pii et cristiani» dilettando il vasto pubblico (le «persone modeste») della poesia. Converrà, a questo punto, offrire il breve elenco, in ordine di apparizione, degli autori riuniti nell'esile miscellanea lirica allegata alla riedizione del I 572 dei Salmi penitentiali:

Antonio Minturno (9), Annibal Caro (3), Pietro Bembo (4), Claudio Tolomei (3), Egidio [da Viterbo] (I) ${ }^{48}$ Federico Fregoso (I), Francesco Petrarca (I), Francesco Maria Molza (3), Francesco Turchi (5), Giovanni Guidiccioni (3), Giovanni della Casa (3), Giovan Francesco Bini (2), Luigi Tansillo (I) ${ }^{49}$

Intorno all'auctoritas petrarchesca, presente con l'immancabile Canzone alla Vergine, Turchi concentra un mannello di poeti di prima grandezza che fa capo a Minturno, a ribadirne il rilievo di letterato e chierico; poi Bembo, Guidiccioni e Della Casa, attestati con una manciata di sonetti che, in linea con le riscritture dei salmi penitenziali, muniti di didascalie che li connotano esplicitamente come invocazioni a Dio e alla Vergine, condividono il tema del pentimento e della conversione. Introdotta da un frontespizio autonomo, l'esile silloge acquista un rilievo cruciale per l'economia complessiva del volume, giacché porta a realizzazione quell'itinerario di conversione e penitenza avviato nei Salmi penitentiali, con i quali forma un corpo unitario. 
La coppia di sonetti di Bembo, O Sol, di cui questo bel sole è raggio $e$ Se già ne l'età mia più verde et calda, cui si aggiunge l'explicit del canzoniere bembiano nella princeps del Is30, la ballata Signor quella pietà che ti constrinse, si articola come preghiera a Dio, in conformità con il modello petrarchesco di Vergine bella, richiamata nella preghiera alla Vergine dell'ultimo sonetto bembiano proposto, Già donna, hor dea, nel cui verginal chiostro. Anche $i$ sonetti dellacasiani trascelti riecheggiano motivi penitenziali, con Io, che l'età solea viver nel fango $e$ Dopo sì lungo error, dopo le tante, culminando con l'«elogio salmodiante dell'attività creatrice di Dio padre», il celebre sonetto Questa vita mortal, che 'n una o 'n due. $^{\text {so }}$

Il tema dell'antagonismo tra poesia amorosa e poesia spirituale e della liceità della combinazione tra discorso lirico e soggetto religioso è messo in luce anche in una preziosa collettanea che si propone al lettore come una «scelta di rime spirituali de' più eccellenti autori d'Italia». Mi riferisco alle Muse sacre, edite nel I608 da Evangelista Deuchino per le cure di Pietro Petracci, un volume in ottavo di 456 pagine che ba il merito di riunire $i$ maggiori poeti attivi tra la fine Cinquecento e il primo decennio del Seicento; la produzione di stampo religioso dei lirici «laici» veniva setacciata a partire dalle raccolte originarie, e dopo diligente selezione le poesie «morali e sacre» venivano ricollocate in un collettore conforme alle aspettative del pubblico di libri spirituali. Nel presentare la silloge, il curatore avvertiva la necessità di affidare a un paratesto introduttivo alcune riflessioni sul principio organizzativo e sulla tipologia della raccolta; nell'avviso ai lettori emergono alcune notizie sul significato e le prospettive della selezione di Petracci, il quale, come avremo modo di appurare, ambisce a rappresentare a tutto tondo il panorama lirico contemporaneo, di cui lo stesso curatore si sente parte integrante, in qualità, non solo di promotore, ma anche di autore:

Quasi tutti gli autori sogliono nel donare alle stampe le loro poesie mescolarne con le amorose delle morali e sacre, le quali volentieri sarebbono tenute e lette da molte sorti di persone religiose, che le schifano per essere unite con suggetti dalla lezzione de' quali pare ad essi di doversene astenere. Onde io mi mossi a raccorne da' più pregiati autori ch'hanno in questa età poste in luce opere poetiche, e con l'aiuto ancora 
di molti che non hanno più stampato ne ordinai questo presente volume con pensiero di formarne il secondo, intorno al quale tuttavia m'affatico. ${ }^{51}$

Anche qui, in piena sintonia con le recenti sillogi d'autore di poesia religiosa orientate alla celebrazione delle disposizioni dottrinali tridentine e delle singole occasioni liturgiche, il principale obiettivo è quello di evidenziare la funzione edificante svolta dall'antologia, grazie alla quale le «persone spirituali et religiose» potevano «passare le ore di ricreazione con lettura non solamente di diletto, ma di utilità meravigliosa), godendo di componimenti incentrati su alcuni tra $i$ concetti cardine del cattolicesimo post-conciliare: "vita e morte del Salvatore, o intorno al martirio di qualche santo, e specialmente intorno all'eccellenze e dolori della Beata Vergine». ${ }^{52}$

Quel che distingue il modello antologico di Petracci rispetto a quelli che lo precedono consiste nel metodo di selezione, che porta allinclusione, accanto a classici volgari e autori di fama ormai consolidata, di poeti emergenti, entrati da poco nelle cronache letterarie. Converrà dunque riportare la rassegna quantitativa, in ordine decrescente, degli autori e dei testi del volume:

Angelo Grillo (64), Giovan Battista Marino (53), Pietro Petracci (30), Torquato Tasso (26), Gabriele Fiamma (23), Giuseppe Policreti (20), Ercole Udine (I 2), Bartolomeo Barco (I 3), Gismondo Santi (I 3), Carlo Coquinato (I4), Tommaso Stigliani (I I), Cesare Rinaldi (I I), Giovanni Ralli (ı), Incerto autore (9), Giuliano Goselini (9), Giacomo Bratteolo (8), Luigi Groto (8), Crisostomo Talenti (8), Gabriello Chiabrera (7), Battista Guarini (6), Giovanni Capponi (6), Gregorio Comanini (6), Filippo Alberti (6), Muzio Manfredi (6), Guido Casoni (5), Marino Nori (5), Vincenzo Giusti (4), Ansaldo Cebà (4), Cesare Orsini (4), Alessandro Gatti (3), Troilo Savorgan (3), Giacomo Antonio Bianchino (3), Alessandro Coperchi (2), Paolin Fiamma (2), Carlo della Serva (2), Tommaso Sabbadini (2), Orazio Susanni (2), Maffeo Venier (2), Il Nesso Academico Trasformato (2), Francesco Panigarola (I), Francesco Rubino (I), Gasparo Murtola (I), Iacopo Sannazaro (I), Giovan Battista Viviani (I), Emilio Barbarossa (I), Pomponio Montanaro (I), Remigio Fiorentino (I), Tiresia Academico Trasformato [Marino Cosentino] (I).

La rassegna indica un insieme abbastanza compatto formato da 47 autori per 434 testi complessivi (9 dei quali di «incerto autore»), dati 
che accentuano il carattere di militanza della collettanea di Petracci, che recluta tra le sua fila $i$ maggiori poeti attivi tra fine cinquecento e inizio seicento, con il primato assegnato ad Angelo Grillo e Giovan Battista Marino - presenti rispettivamente con 64 e 53 testi, il $27 \%$ di quelli totali-, campioni assoluti di questo nuovo canone della lirica spirituale di epicentro veneziano. La trafila degli autori contemporanei (Chiabrera, Guarini, Stigliani, Rinaldi, Capponi, ecc.) si sourappone al quadro d'insieme della poesia della seconda metà del Cinquecento, miscelando in modo armonico chierici e laici; prendono parte i poeti più celebri attivi nel primo decennio del Seicento, dei quali il curatore vuole dimostrare la capacità di saper verseggiare anche su tematiche «oneste». Le modalità di selezione e formazione della raccolta si spiegano dunque entro $i$ termini di un giudizio critico-letterario proiettato a tutto campo, pronunciato con l'intento di inserirsi nel dibattito lirico coevo, profilando una nuova canonizzazione della poesia sacra sensibile alle novità introdotte, in termini di autori e poetiche, a cavallo dei due secoli. Una canonizzazione, si badi, su cui pesano le conoscenze e le relazioni del curatore, che sfrutta l'antologia come mezzo di promozione di sé (in evidenza nel rilievo assegnato alla propria produzione in versi con ben 30 testi antologizzati) e dei suoi sodali più stretti. La palma conferita a Grillo, del quale Petracci fu amico e collaboratore, curandone $i$ volumi delle Lettere stampati nel I608 e I6I2 e stendendo gli argomenti ai Pietosi affetti, è eloquente, testimoniando non solo l'importanza cosi rapidamente raggiunta dal benedettino ligure nel quadro della poesia religiosa ma anche il prestigio intellettuale conquistatosi negli ambienti editoriali e culturali veneziani. ${ }^{33}$

Grillo e Marino sembrano dunque spartirsi l'onore di rappresentare il genere della lirica spirituale primosecentesca ma, quantunque la loro supremazia non sia discutibile e sia stata anzi confermata dagli studi critici più recenti, nella scelta di Petracci dovettero pesare pure ragioni di mercato e convenienza personale, considerato il vincolo professionale che lo teneva proprio in quegli anni legato, in qualità di consulente $e$ redattore, agli stampatori veneziani Ciotti e Deuchino, che, com'è noto, licenziarono alcune importanti opere di Grillo e Marino. ${ }^{54}$

L'esame, operato a campioni, concernente la derivazione dei testi, indica che Petracci si servi quasi esclusivamente di raccolte individuali uscite in quegli anni a Venezia, invertendo cosi una tendenza che aveva visto $i$ redattori procurarsi $i$ testi in larga parte da antologie a stampa. Dalle 
edizioni Ciotti dei Pietosi affetti di Grillo e delle Rime mariniane, datate rispettivamente I60I e I602, Petracci seleziona $i$ testi dei due campioni incontrastati della miscellanea. Sul fronte mariniano si segnala la predilezione assoluta del curatore per la seconda parte di Madriali e canzoni, da cui è tratto il maggior numero dei componimenti sacro-morali, nello specifico madrigali; interessante notare come dei 4I sonetti presenti nella partizione delle rime sacre della raccolta mariniana Petracci ne selezioni soltanto due, Quando Cerere in Cristo udì Natura $e$ Se di tante bellezze adorno e pieno, scelta che si puo motivare con il tentativo di allineare le forme della poesia sacra del Marino ai registri stilistico-formali dei Pietosi affetti di Grillo, nel quadro di una lirica spirituale declinata in chiave melica e madrigalesca. ${ }^{55}$

Ma anche altri corpora testuali, tra quelli quantitativamente più rappresentativi provengono dagli annali tipografici di Ciotti: ${ }^{6}{ }^{i}$ I $_{3}$ madrigali di Bartolomeo Barco derivano dall'edizione dei suoi Madrigali del 1604, una raccolta che, a dispetto del titolo generico, è dedicata quasi integralmente alle figure di Cristo e della Vergine, tra gli architravi tematici, lo si è detto, delle Muse sacre. Discorso simile va fatto per $i$ Cento madrigali sopra la B. Vergine Maria di Giacomo Ralli, editi da Ciotti per le cure dello stesso Petracci, il quale riversa nell'antologia Io madrigali. Ancora: $i$ componimenti di Guarini provengono dall'edizione Ciotti delle Rime (I598) come pure quelli dell'accademico Insensato di Perugia Filippo Alberti, autore di una raccolta poetica edita nel I602, ${ }^{57}$ mentre gli II componimenti di Tommaso Stigliani sono ricavati dalla sesta parte, dedicata ai «soggetti moralis delle sue Rime, pubblicate sempre da Ciotti nel I60s. Dai prodotti dell'editoria veneziana Petracci ricava sia $i$ testi di Chiabrera, corrispondenti al primo blocco di sette canzoni edite nel volume delle Rime sacre $[. .$.$] novamente poste in luce per i$ tipi di Sebastiano Combi nel I605, sia i I4 madrigali di Carlo Coquinato, apparsi in un libro di Madrigali uscito presso Deuchino nel I600, sia gli II componimenti del bolognese Gelato Cesare Rinaldi, accolti nell'edizione Zanetti delle Rime (I605). Significativa, infine, risulta il reperimento di alcuni componimenti di rimatori udinesi, operato pescando soprattutto dalle Rime di diversi elevati ingegni de la città di Udine, ${ }^{58}$ curata da Giacomo Bratteolo, presente qui con 8 testi, scelta che si spiega con le origini friulane di Petracci, che infonde così una sfumatura 
personale, di orgoglioso provincialismo, a una silloge che si rivolge a una platea nazionale.

Da questa scorsa sommaria delle fonti testuali si evince come fosse pressante per Petracci la necessità di dare voce soprattutto ad autori contemporanei, conferendo pieno diritto di cittadinanza ad alcuni tra $i$ maggiori rimatori immessi di recente sul mercato dell'editoria veneziana e selezionando quanto di «spirituale» era stato confusamente pubblicato sino a quel momento. Tuttavia, benché la silloge voglia dare innanzi tutto visibilità alle nuove tendenze della produzione lirica di carattere sacro si segnalano alcuni significativi inserimenti relativi al piu recente passato, che offrono un canone innovativo rispetto a quello fornito dai volumi antologici editi nella seconda metà del Cinquecento, schiacciati su autori attivi prevalentemente nella prima metà del XVI secolo. Appare dunque rilevante trovare antologizzati due nomi di punta della lirica spirituale di secondo Cinquecento come Gabriele Fiamma e Torquato Tasso, a testimoniare nitidamente l'evoluzione stilistica e tematica della lirica di argomento religioso, che nel secondo Cinquecento aveva esteso $i$ suoi ambiti oltre il consueto esercizio di spiritualizzazione del repertorio petrarchesco e conseguentemente accentuato le funzioni celebrative, evocando persone, feste e simboli della nuova cultura religiosa affermatasi al termine del Concilio di Trento. I 26 componimenti tassiani derivano compattamente dalla silloge delle Rime spirituali edite a Bergamo da Comin Ventura nel I597, di cui Petracci recupera sia la seriazione sia le disdascalie, escludendo però i testi maggiormente vincolati a una retorica encomiastica. I 23 testi di Fiamma sono invece verosimilmente tratti dalla ristampa di Ciotti delle Rime spirituali del I608, pubblicate anche qui con gli argomenti di Petracci, che nella dedica ad Antonio Grimani assegnava un primato alle «poesie spirituali» del Vescovo di Chioggia, ritenendo che «in si fatto genere sono delle principali che si leggano». ${ }^{59}$ Il mélange tra rimatori in attività e nuovi classici della poesia volgare configura un insieme coerente e omogeneo, a testimoniare la solidita di un progetto antologico nato da un orientamento critico volto a caldeggiare le voci del nuovo Parnaso spirituale secentesco. Si avverte, dunque, il proposito da parte di Petracci di fondare, in ottica militante, un nuovo canone della lirica spirituale, à la page e proiettato verso il futuro, capace di scalzare quello di orbita primocinquecentesca diffuso nelle antologie precedenti. Nel novero degli autori, infatti, troviamo emblematicamente assenti $i$ nomi della 
Colonna e di Malipiero, che avevano invece monopolizzato la miscellanea veneziana di Rime spirituali del I550-I552, a segnalare un avvicendamento di modelli che bene rispecchia l'evoluzione della lirica spirituale in volgare. L'impresa di Petracci testimonia dunque una nuova fase di canonizzazione della poesia religiosa, riaffermando un'esigenza da sempre costitutiva delle antologie liriche, ossia quella di documentare, in sintonia con le tendenze imposte dalle politiche editoriali, la vitalità di una nuova generazione di poeti da promuovere ed elevare, talvolta, al rango di modelli. In questo senso va rimarcato il ruolo ricoperto dalle Muse sacre nella definizione di un'idea di lirica marcata dalla sperimentazione, che riconosce al Marino un rilievo indiscusso anche nel campo della poesia di argomento religioso, a dispetto della vena prioritariamente «erotica» della sua produzione. ${ }^{60}$

Una nota conclusiva relativa all'impiego dei metri: le scelte di Petraci rivelano un'apertura verso forme in ascesa nel panorama secentesco, come il madrigale e la canzonetta, che predominano nelle partizioni dedicate ai due «primatisti» della raccolta, Grillo e Marino, mettendo in discussione la tradizionale egemonia del sonetto.

Germogliate sul modello delle Muse sacre sono le due parti del Nuovo concerto di rime sacre, tutte ripiene di bellissimi et esquisiti concetti composte da' più eccellenti poeti d'Italia, edite a Venezia presso Antonio Pinelli nel $1616 .{ }^{61}$ La raccolta veniva allestita dal veneziano Eugenio Petrelli, che nella dedica della prima parte indirizzata al doge di Venezia Giovanni Bembo affermava di aver «raccolto e ridotto in concerto le rime sacre de' più valorosi poeti de' nostri tempi, nelle quali con spiegatura ammirabile si sono messi a lodar Dio, la Regina del Cielo sua Madre, e li santi, e con cristiana moralità ad eccitar le pie menti alla contemplazione del Celeste Regno». ${ }^{62} \mathrm{La}$ selezione degli autori e, dunque, il numero dei rispettivi testi editi nella prima parte rispecchia una graduatoria allora indiscussa negli ambienti letterari veneziani del secondo decennio del Seicento; come nelle Muse sacre, la coppia Grillo-Marino, seguita a ruota da Tasso, conferma un primato, salendo stabilmente ai vertici del canone della lirica spirituale. Al contempo, conta rimarcare il rilievo dato a due chierici locali come Crisostomo Talenti e Maurizio Moro, il primo presente con 20 unità il secondo con I8, in prevalenza madrigali, ospitati interamente nella prima parte. A destare un certo interesse è però l'innesto di autori esordienti, da poco apparsi sulla scena editoriale: il caso più rilevante è 
quello del salentino Antonio Bruni, presente con 36 testi riconducibili alla sua Selva di Parnaso, primo di una serie di libri di rime che lo renderà celebre nel panorama secentesco. ${ }^{63}$ Sul piano dell'assetto strutturale, la raccolta è suddivisa in partizioni tematiche, nelle quali sono concentrate le rime dei vari autori, afferenti ai macrotemi di Cristo e della Vergine, che dominano, come segnala il frontespizio, la prima parte della raccolta.

Una tappa importante di questo percorso di antologizzazione della lirica spirituale in volgare è costituita da una monumentale raccolta allestita da Mauriżio Di Gregorio, il Rosario dalle stampe de tutti i poeti e poetesse, antichi e moderni, cinquecento di numero (Napoli I6I4), presentato come l'ottavo tomo di un Giardino di tutte le scientie. Con il Rosario il frate domenicano dava alla luce un prodotto sostanzialmente nuovo per struttura e ampiezza dei dati raccolti al suo interno, concepito per la vita devozionale e religiosa stricto sensu. Allestita in forma enciclopedica, l'antologia di Di Gregorio risulta straordinaria per numero degli autori coinvolti e per il carattere di militanza con cui fu realizzata, a testimoniare la grande attenzione prestata dal curatore per la lirica come strumento di catechesi ed educazione spirituale, nell'ottica di un'opposizione totale agli usi e ai contenuti della poesia amorosa, travalicante nel peccato d'idolatria: «Alcuni poeti, per non aver posto nelle stampe rime spirituali, imitando il mal Imperio che l'amor della Signora Laura in Cristo lo trasmutò [...]». ${ }^{64}$ Sono parole, queste, tratte dalla premessa dell'Autore a i Momoisti collocata in apertura dell'edizione, che consente di dare una precisa valutazione ideologico-culturale del progetto editoriale e, al contempo, di approfondire i criteri in base ai quali Di Gregorio raccolse $i$ testi destinati all'antologia, rilevando pure le sostanziali differenze rispetto agli altri modelli antologici. Nella premessa ai lettori emerge un altro passaggio degno di attenzione, che colloca l'operazione nell'alveo dell'ortodossia tridentina:

Sappiate che fedelmente son recitati i poeti, e con ortografie antiche, eccetto che alcuna parola qual posseva aver senso ereticale, come in quella di D. Vittoria Colonna del Corpo di Cristo, chiamandolo Corpo celeste, che si può ridurre alle eresie di Valentino, che poneva il Corpo di Cristo essere celeste, l'ho mutato in puro, e così de gl'altri simili, toccando a' teologi di struger l'eresie, come sopra le sentenze e nella gionta al Conc. di Trento. 
La censura ai versi della Colonna, che attraverso il richiamo al docetismo valentiniano, toccava uno dei cardini della controversia antiprotestante come il culto eucaristico, si estendeva anche ad altri passi poetici ritenuti non conformi ai decreti conciliari, nelle forme di un supporto strategico agli obiettivi dei «teologi», al fine di tutelare l'uniformità del messaggio dottrinale sotteso ai testi poetici. Tuttavia, la mappatura della lirica religiosa operata da Di Gregorio è il frutto di una selezione di materiali disparati, orientata a presentare quanti più autori possibili, scevra, quindi, dalla volontà di fornire un canone antologico: «Per la copia de' poeti non ho tessuto ordine di nobiltà, né di eccellenza di verso, ma solo chi prima mi capitava ponevo e secondo il titolo che nell'opre ho trovato ho posto». ${ }^{65}$ Inutile in questo caso ragionare sulle presenze e sulle assenze, visto che il Racconto de i poeti dell'opera posto in coda al volume pullula di 500 nomi elencati in ordine alfabetico, configurando, più che un florilegio, un vero e proprio repertorio della lirica spirituale. Una silloge che si schiera, secondo uno schema ormai topico, in aperta concorrenza con la tradizione delle miscellanee profane, che Di Gregorio dimostra di conoscere bene nella loro articolazione editoriale in un lungo regesto che occupa quasi una pagina, dove sono citati $i$ curatori di alcune tra le maggiori tipologie antologiche apparse fino ad allora:

Che se il Dolce, il Roscelli, il Domenichi, il Gentile, il Moboni, il Contile son lodati per aver raunati pochi poeti, come scelta di rime, muse toscane, fiori di rime, rime degne, corona d'Apollo, ghirlanda di poeti, fiori di poeti, garriggiamento poetico, le donne illustri, il tempio di Donna Giovanna d'Aragona, le nove Muse, Parnaso di poeti, l'ingegni, Elicona di poeti, giardino di poeti, raccolta de funebri di tanti pontefici, di tanti reggi e regine, del Signore Cardinal Alessandro Farnese, del Marchese del Vasto, raccolta di rime di Cavalieri napolitani, concetti poetici $\left[. . .{ }^{66}\right.$

Da questo rapido esame dei paratesti introduttivi appare dunque evidente come il Rosario abbia la pretesa di configurarsi come la principale via d'accesso devota e pastorale alla forma-antologia, una vera e propria enciclopedia della tradizione lirico-spirituale costituita da preghiere in versi che devono stimolare la meditazione del fedele. ${ }^{67}$ Anche l'assetto del volume ricalca la ripartizione liturgica del rosario in tre corone corrispondenti ai misteri gaudiosi, dolorosi e gloriosi. Nelle Is sezioni di cui si compone la raccolta, comprese tra l'Annunciazione 
dell'Arcangelo Gabriele e l'incoronazione di Maria, sono riversati quanti più materiali possibili relativi alla meditazione sui misteri della vita di Cristo e della Vergine, un affastellamento quasi del tutto casuale di autori e componimenti (o singoli brani testuali) mescolati in maniera caotica e riproposti nel corso del volume senza alcuna distinzione, entro un edificio dove non conta la personalità dei singoli autori ma la sistemazione enciclopedica di un universo testuale disarticolato, che il curatore ha faticosamente compendiato e perimetrato entro $i$ margini del consueto libricino da tasca.

Se nella seconda metà del Cinquecento alle raccolte di rime spirituali di diversi si affiancano sillogi allestite sulla base di affinità metriche e retoriche - si pensi ai vari laudari, ai summenzionati Salmi penitentiali di diversi eccellenti autori $e$ alla Nuova raccolta di lagrime di più poeti illustri, edita a Bergamo nel I593, che accoglie alcune tra le prove piu alte del registro lacrimoso ad opera di Torquato Tasso, Erasmo di Valvasone e Angelo Grillo ${ }^{68}$-, nei primi decenni del Seicento si assiste a un insistita specializzazione tematica del formato antologico e alla conseguente fioritura di miscellanee riservate a determinati settori liturgico-devozionali.

La spiritualità francescana emerge in tutta la sua esemplarità nelle Rime spirituali di diversi autori in lode del serafico Padre S. Francesco, e del sacro Monte della Verna, edite a Firenze nel I606 per le cure del frate Silvestro da Poppi, che attua una selezione testuale attentamente meditata nelle sue scansioni interne, che ai tre canti in ottave della Vita del Serafico et glorioso S. Francesco di Lucrezia Marinella e al Capitolo sopra la vita e stimmate del glorioso padre S. Francesco del frate Giovanni da Stia congiunge un'esile sezione di Sonetti di diversi in lode di S. Francesco che ospita due sonetti di Tasso e quattro madrigali di Marino, a riprova di come $i$ due autori fossero ormai entrati a pieno titolo nel canone della poesia spirituale. ${ }^{69}$

Un secondo esempio di questa tendenza delle antologie di poesia religiosa a rivolgersi a un versante specifico della predicazione religiosa è rappresentato da La celeste lira - licenziata a Venezia da Deuchino nel I6r2 e allestita ancora da Petracci-, che il sottotitolo del frontespizio designa come «componimenti di diversi eccellentissimi autori sopra il Santissimo Sacramento dell'Eucarestia». Uno dei maggiori capisaldi 
della retorica conciliare e antiluterana diventava il perno di una silloge che miscelava autori laici di larga fama a più oscure figure di chierici locali. Rispetto alle gerarchie prodotte nella più varia e ambiziosa raccolta delle Muse sacre, Petracci piega il metodo di selezione all'esigenza primaria di rispettare il campo tematico, selezionando da volumi già editi soltanto $i$ testi coerenti con l'indirizzo prescelto. Tale limitazione argomentativa non può non avere una ricaduta sulle graduatorie autoriali, e dunque sull'assetto qualitativo dell'antologia; se Tasso, Grillo e Marino raggiungono in totale I2 testi, a conquistarsi un primato assoluto è il «canonico secolare» di San Giorgio in Alga Maurizio Moro, che con i suoi 43 componimenti (in maggioranza madrigali) esibisce tutto il rilievo conquistatosi come autore di raccolte madrigalesche di stampo spirituale.

Un altro ramo della specializzazione delle antologie di lirica religiosa è rappresentato da quelle raccolte che tra la fine del Cinquecento e la prima metà del Seicento declinarono in chiave sacra la tipologia profana del tempio di rime in onore di uomini e donne illustri. ${ }^{70}$ Si tratta di sillogi che, trasferendo sul terreno spirituale una moda letteraria di grande impatto socio-mondano, riuniscono testi di diversi autori che avevano versificato intorno ai principali culti della devozione cattolica. Il filone mariano caratterizza il Tempio armonico della beatissima Vergine (Roma I599) allestito da Giovanni Giovenale Ancina, una silloge di laudi e altre forme «cantabili» fiorita in seno all'Oratorio di Filippo Neri che, pur ponendosi ai margini dei circuiti ufficiali della lirica, testimonia un'assimilazione profonda della lezione petrarchesca, componente fondamentale di tutto il repertorio laudistico coevo. ${ }^{7 \mathrm{I}}$ L'esame dei I20 componimenti accolti nel volume rivela un profondo lavoro di riscrittura svolto dal curatore, che ritocca anche pesantemente alcuni testi "profani» allo scopo di spiritualizzarne il messaggio e renderlo conforme alla nuova destinazione liturgica. Nella lettera di dedica al Maestro del Sacro Palazzo Giovanni Maria Guanzelli, Ancina afferma esplicitamente di aver atteso «alla riforma dell'opre profane e lascive, tramutandole in spirituali, serbandosi però nella sua prima integrità la soave e dolce armonia, tanto da' gentili spiriti bramata e desideratas $\rangle^{72}$; dunque, come segnala un altro dei paratesti introduttivi che arricchiscono di preziose indicazioni di metodo l'edizione del I599, il Tempio riunisce 
diverse composizioni più vaghe, ariose e scelte di diversi autori de' più rari, celebri, migliori e più esquisiti de' nostri tempi, antichi e moderni, spirituali e profani; e di questi ultimi, toltene via le parole vane e profane, sopra l'istessa lor musica di prima, poco o niente alterata, stesovi altre parole buone, oneste, divote e pie, acciò che, in tal guisa accomodate, puotesse ognuno, eziamdio religioso o santo, libera e sicuramente, senza scrupolo e fuor di scandalo, goder della suddetta armonia, et onestamente trastullandosi ricrearsi in quella. ${ }^{73}$

Per chiarire la logica strutturale e la ratio organizzativa dell'impresa di Ancina risulta di grande interesse anche la lettera indirizzata a Geronima Colonna d'Aragona, dedicataria, com'è noto, di un celebre Tempio, edito a Padova nel is68 per iniziativa del napoletano di nascita e padovano di formazione Ottavio Sammarco. ${ }^{74}$ Conviene riportarne un ampio passaggio:

Già trentatré anni passati, ritruovandomi nel famoso e celebre studio di Padova, per mia buona sorte vi conobbi un cavalier napolitano detto per nome Ottavio Sanmarco, gentilissimo invero e molto spiritoso signore, il quale con grandissimo affetto di cuore ed esattissima diligenza tenea per impresa l'andar raccogliendo molte e varie composizioni de' poeti più eccellenti e rari di quel tempo, esquisite e scelte, per edificarne un Tempio a Vostra Eminenza Illustrissima, che poi di là a poco egli mandò fuori in luce intitolato Tempio della Colonna, ad imitazione e a gara d'un altro primiero detto il Tempio dell'Aragona. Da indi in qua, essendo antiquati e poco men che caduti e ormai, si può dire, passati in oblivione l'uno e l'altro di questi, ecco sorta opportuna occasione di fabricarne un altro nuovo e vistoso, ma molto differente da quelli, e di tanto a loro superiore quanto le cose divine all'umane e le celesti alle terrene prevagliono e per ogni verso con debita ragione s'antipongono. [...] Dove all'incontro questo nuovo Tempio sorgente vien dedicato a donna immortale, incorottibile, tutta pura, bella e santa, senza macchia veruna, anzi fu già per grazia impeccabile e or gloriosa e trionfante in Cielo: ed è questa la beatissima Vergine, Madre di Dio, Regina de' Cieli, padrona degli angeli, gran Signora e Avvocata nostra. Ordunque, a questa sì che si possono e devono fabricarsi ed erigersi tempî per lodarla, per celebrarla, esaltarla e magnificarla a più puotere, senza scropolo di conscienza e senza correr pericolo d'adulazione né di soverchia laude iperbolica; imperoché quanto più ella si loda, tanto più si rende e scuopre lodevole, né di lei manca giamai nuova materia di lode, ove non manchi copia di lodatore. Di cui 
tutto il contrario avviene in que' tempî che in donne viatrici, ancor viventi e mortali, per grandi e sublimi che siano, communemente fabricar e dedicar si sogliono, cioè che per lo più si scorgono pieni di leggerezza e vanità mondana, di ciarle, di menzogne, di lascivia umana, mescolata però con qualche parte d'onestà sotto colore di poetica vaghezza e leggiadria; colmi d'adulazione, e finalmente poi ridondanti di ridicole e vergognose, per non dire vituperose, o forsi anco talor scandalose iperboli e superstiziose, in quella parte ove spirano odor d'idolatria, attribuendo quel ch'a Dio ed a' suoi santi dirittamente si conviene, e distorcendolo a creature corrottibili e vane, sottoposte a mille miserie fin alla morte. ${ }^{75}$

Collocando al centro dell'edificio encomiastico la Vergine nella sua varia pluralità di immagini e occasioni liturgiche, l'obiettivo di Ancina era quello di convertire significato e valore ideologico della forma-tempio offrendone un'alternativa speculare, capace di opporsi all' «idolatria» che serpeggiava nelle antologie profane. Ci troviamo davanti, dunque, a una raccolta che, facendo leva sulla forza attrattiva del culto mariano, concorreva al definitivo radicamento, nel mercato del libro di poesia in volgare, della lirica spirituale, presente ormai con una sua precisa fisionomia culturale, simmetrica a quella profana, e con una sua consolidata trafila di autori (e testi) di riferimento. Ne è prova anche, tornando agli inizi del Seicento, il Sacro tempio dell'imperatrice de' Cieli Maria Vergine Santissima (I6I3), una raccolta di poesie mariane, scrupolosamente ordinate per capi, che conferma in buona sostanza il canone primosecentesco della lirica religiosa, dove primeggia il solito Grillo (48 testi), seguito a distanza da Fiamma (I5), Marino (13), Tasso (8) e da alcuni tra i più celebri poeti che avevano calcato almeno una volta il sacro Parnaso. ${ }^{76}$

Pietro Giulio Riga 
Pietro Giulio Riga

I. Sull'importanza delle antologie nella cotruzione di un canone lirico rimando ad Amedeo Quondam, Petrarchismo mediato. Per una critica della forma «antologia», Roma, Bulzoni, I 974; Luise George Clubb - William George Clubb, Building a Lyric Canon: Gabriel Giolito and the Rival Antologists I545-I590, "Italica», LXVIII (I99I), pp. 332-344. Sulle funzioni e sulla struttura delle miscellanee di poesia lirica si veda Amadeo Quondam, Il naso di Laura. Lingua e poesia lirica nella tradizione del Classicismo, Modena, Panini, i99i, pp. i 81-199; Maria Luisa Cerrón Puga, Materiales para la construcción del canon petrarquista: las antologías de «Rime» (libri I-IX), «Critica del testo», II (I 999), pp. 259-290; si vedano poi i saggi raccolti in Monica Bianco ed Elena Strada (a cura di), «I più vaghi e i più soavi fiori». Studi sulle antologie di lirica del Cinquecento, Alessandria, Edizioni dell'Orso, 200 I, in particolare Franco Tomasi, Alcuni aspetti delle antologie liriche del secondo Cinquecento (ibid., pp. 77-I I I) e Paolo Zaja, Intorno alle antologie. Testi e paratesti in alcune raccolte di lirica cinquecentesche (ibid., pp. I I 3-145). Di grande utilità, per la mappatura del fenomeno delle antologie liriche a stampa del XVI secolo, il progetto Lyra (http://lyra.unil.ch), che fornisce descrizioni dettagliate di alcuni importanti volumi, con indicazione sul contenuto dei testi, permettendo di operare indagini comparative. Sul concetto e sulla pratica del modello antologico rimando ai contributi raccolti in Antologie, a cura di Bianca Maria Da Rif e Silvio Ramat, Padova, Il Poligrafo, 2009, e in Enrico Malato e Andrea Mazzucchi (a cura di), Antologie d'autore. La tradizione dei florilegi nella letteratura italiana. Atti del Convegno internazionale di Roma, 27-29 ottobre 2014, Roma, Salerno Editrice, 2016.

2. Se ne veda l'edizione moderna: Rime diverse di molti eccellentissimi autori (Giolito I545), a cura di Franco Tomasi e Paolo Zaja, Torino, Edizioni RES, 200 r. Ricordo che la serie delle Rime diverse si chiuderà nel i 560 con la pubblicazione di un Libro nono. Sulle antologie liriche licenziate dalla Fenice rinvio a Angela NuovoChristian Coppens, I Giolito e la stampa nell'Italia del XVI secolo, Genève, Droz, 2005 , pp. IIO-II 5 .

3. Alle pubblicazioni a stampa si associa ancora il fenomeno delle miscellanee manoscritte di poesia lirica ad uso privato o semiprivato; in proposito si veda il contributo di Simone Albonico, Antologie di lirica cinquecentesca, in Antologie d'autore, cit., pp. 173-206. Sul successo delle antologie liriche nella seconda metà del Cinquecento si veda il quadro riassuntivo di Amedeo Quondam, Il pubblico della poesia, in Sergio Luzzatto e Gabriele Pedullà (a cura di), Atlante della letteratura italiana. II. Dalla Controriforma alla Restaurazione, a cura di Erminia Irace, Torino, Einaudi, 201 I, pp. 79-86.

4. Un sussidio fondamentale alla mia ricerca proviene dagli studi che negli ultimi anni si sono concentrati sulla lirica di argomento religioso, a partire dalla mappatura di Amedeo Quondam, Note sulla tradizione della poesia spirituale e religiosa (parte prima), con l'aggiunta di un Saggio di bibliografia della poesia spirituale (I470-1600), in Paradigmi e tradizioni, a cura di Id., Roma, Bulzoni, 2005, pp. I 27-282. Si veda anche Stefano Carrai, La lirica spirituale del Cinquecento, nel suo L'usignolo di Bembo. Un'idea della lirica italiana del Rinascimento, Roma, Carocci, 2006, pp. I 23 -1 35. Importanti indicazioni nei saggi contenuti in Poesia e retorica del Sacro tra Cinque e Seicento, a cura di Erminia Ardissino ed Elisabetta Selmi, Alessandria, Edizioni dell'Orso, 2009, e in due volumi della Collana di studi della Fondazione Pellegrino curati da Maria Luisa Doglio e Carlo Delcorno: Rime sacre dal Petrarca al Tasso, Bologna, Il Mulino, 2005 e Rime sacre tra Cinquecento e Seicento, Bologna, Il 
Mulino, 2007. Utile soprattutto sul piano della valutazione formale della lirica spirituale lo studio di Marc Föcking, Rime sacre und die genese des barocken Stils. Untersuchungen zur Stilgeschichte geistlicher Lyrik in Italien 1536-I6I4, Stuttgart, Steiner, I 994 .

5. Cfr. Ginetta Auzzas, Notizie su una miscellanea veneta di rime spirituali, in Rime sacre dal Petrarca al Tasso, cit., pp. 205-220; Quondam, Note sulla tradizione, cit., pp. I 8 I-I 83; Matteo Fadini, L’inquietudine in versi. Le opere di Marcantonio Cinu₹zi e la letteratura religiosa eterodossa. Tesi di dottorato di ricerca in Studi letterari, linguistici, filologici dell'Università degli Studi di Trento (tutor: Andrea Comboni), XXVI ciclo, Anno Accademico 201 2-2013, pp. 75-I I 2.

6. Cfr. Auzzas, Notizie su una miscellanea veneta, cit., p. 208.

7. In proposito cfr. Danilo Zardin, L'arte dell'apprendere «soave». Poesie e canti religiosi nell'Italia del Cinque-Seicento, in Giancarlo Rostirolla, Danilo Zardin e Oscar Mischiati, La lauda spirituale tra Cinque e Seicento. Poesie e canti devozionali nell'Italia della Controriforma, Roma, Ibimus, $200 \mathrm{I}$, pp. 695-739.

8. Iacopone da Todi, Laude, in Venetia, nella contrada di Santa Maria Formosa al Segno della Speranza, I 556; Laude devote composte da diverse persone spirituali, in Venetia, al Segno della Speranza, I 556; cfr. La Biblioteca volgare I. Libri di poesia, a cura di Italo Pantani, Milano, Editrice Bibliografica, I996, p. I 59 num. 2565 e p. 167 num. 2697.

9. Ho consultato l'esemplare della Biblioteca Apostolica Vaticana, segnato Ferraioli VI 460.

ı. Libro primo delle rime spirituali, in Venetia, al Segno della Speranza, i 550 , cc. $49^{r-104 v}$. Sul capitolo della Colonna $c f r$. Antonio Corsaro, Fortuna e imitazione nel Cinquecento, in Claudia Berra (a cura di), I Triumphi di Francesco Petrarca, Bologna, Cisalpino, 1999, pp. 429-503, in particolare pp. 465-469; Rosa Casapullo, Per una lettura del Trionfo di Cristo di Vittoria Colonna, in Gabriella Alfieri (a cura di), Storia della lingua e storia, Firenze, Cesati, 2003, pp. 337-355.

I I. La sezione di Malipiero è così intitolata Sonetti di M. Francesco Petrarca divenuto theologo et spirituale per gratia di Dio, et studio di F. Hieronimo Maripetro Minoritano (Libro primo, cit., cc. I05 $r$ - I $84 r$ ); alla c. I $84 v$ compare un sonetto di Malipero con la rubrica Rifugio al clementissimo Signore Iesu Christo nelli deliri affanni della presente vita (inc.: A te IESU confugge il mesto core).

I 2. Cfr. Tomasi, Alcuni aspetti delle antologie liriche, cit., p. Io I e n.

I 3. La distribuzione presenta infatti 20 autori con un solo sonetto e i 2 con due; nel Libro primo, dunque, quello a più alta densità di presenze, il 68\% degli autori si attesta in una fascia compresa tra I e 2 sonetti, a riprova di come l'esigenza di accumulare testi abbia influenzato in profondità l'intero progetto redazionale.

I4. Sulla funzione culturale svolta dalla riscrittura petrarchesca di Malipiero rinvio ad Amedeo Quondam, Riscrittura, citazione e parodia. Il Petrarca spirituale di 
Girolamo Malipiero, in Id., Il naso di Laura, cit., pp. 203-262. La Colonna fu designata da Gabriele Fiamma come la prima ad aver adattato i temi della letteratura devozionale all'esercizio lirico: «Et certamente che, essendo noto a ciascuno che l'Illustrissima Signora Vittoria Colonna, Marchesa di Pescara, è stata la prima ch'ha cominciato a scrivere con dignità in rime le cose spirituali, e m'ha fatta la strada et aperto il camino di penetrare e giungere ove è piaciuto a Dio di condurmi» (Gabriele Fiamma, Rime spirituali [...] esposte da lui medesimo, in Vinegia, presso Francesco de' Franceschi senese, I 570, dalla lettera di dedica a Marcantonio Colonna, c. 4r). Sull'opera di Fiamma, ispirata alla «leggiadra gravità» dei Salmi, si rimanda al saggio di Paolo Zaja, "Perch'arda meco del tuo amore il mondo». Lettura delle Rime spirituali di Gabriele Fiamma, in Poesia e retorica del Sacro, cit., pp. $235-292$.

I 5. Cfr. Franco Tomasi, Letteratura tra devozione e catechesi: il caso di Giovanni Del Bene (I5I3-I559), in Poesia e retorica del Sacro, cit., pp. 55-103. Su Torti un fugace accenno in Auzzas, Notizie su una miscellanea, cit., p. 2 I 2 n.

i6. Sulla componente spirituale presente nel Canzoniere petrarchesco, soprattutto in relazione al tema della preghiera, fa luce il saggio di Lorenzo Geri, «Dopo $i$ perduti giorni». La preghiera nei Rerum vulgarium fragmenta, «Petrarchesca», 5 (2017), pp. 2I-37. Sulla rielaborazione in senso religioso del modello petrarchesco si veda anche Giorgio Forni, Vittoria Colonna, la «Canzone alla Vergine» e la poesia spirituale, in Rime sacre dal Petrarca al Tasso, cit., pp. 63-94.

17. Si allude al trittico $O$ messaggier di Dio, che 'n bigia vesta, A quei ferventi spirti, a le parole e $O$ sante figlie de l'eterno Sire (Libro primo, cit., cc. I $5 v$ - I $6 r$ ). Per approfondimenti testuali rimando a Giovanni Guidiccioni, Rime, edizione critica a cura di Emilio Torchio, Bologna, Commissione per i testi di lingua, 2006, pp. 76-78 e 202-204. Da sottolineare che nel Primo libro delle rime scelte da diversi autori, di nuovo corrette et ristampate (Venezia, Giolito, I 563 ) il sonetto O messaggier di Dio è accompagnato dalla didascalia «Scritto a Fra Bernardino Ochino, alhora catholico». Per un profilo dell'Ochino e per ulteriori ragguagli bibliografici mi limito a rinviare alla voce di Miguel Gotor, Ochino, Bernardino, in Dižionario Biografico degli Italiani, vol. LXXIX, Roma, Istituto dell'Enciclopedia italiana, 2013 3, pp. 90-97.

18. Libro primo delle laudi spirituali da diversi eccellenti e divoti autori, antichi e moderni, composte, in Venetia, ad instantia de' Giunti di Firenze, I 563. Razzi fu autore in proprio di una raccola di laudi intitolata Santuario di laudi, o vero rime spirituali per le feste di ciaschedun santo, in Firenze, appresso Bartolomeo Sermartelli, I 609, nella cui dedicatoria a Suor Vittoria Malespina si menziona l'antologia laudistica del i 563 . Per un profilo biografico e culturale di Razzi, con relative indicazioni bibliografiche, si veda Serafino Razzi, Vita di Santa Caterina de' Ricci con documenti inediti antecedenti l'edizzione, a cura di Guglielmo M. Di Agresti, Firenze, Olschki, I965, pp. XVII-CXXXV.

I9. Sulla categoria socialmente articolata dei «semplici», rivolti alla preghiera in volgare, rinvio a Gigliola Fragnito, Proibito capire. La Chiesa e il volgare nella prima età moderna, Bologna, Il Mulino, 2005, pp. 26 I-274.

20. Cito dal frontespizio del Libro primo delle laudi spirituali, che leggo nell'edizione anastatica di Bologna, Forni, I969. 
2I. Il genere godette di svariate raccolte individuali e collettive; $c f r$. Oscar Mischiati-Giancarlo Rostirolla, Per una bibliografia delle fonti a stampa della Lauda post-tridentina (I563-1952), in La lauda spirituale, cit., pp. 742-784.

22. Sulla ricezione delle Laude di Iacopone da Todi tra Cinque e Seicento rimando a Giacomo Jori, Tradition des imprimés et lectures de Jacopone aux XVI et XVII siècles, in François Trémolières (éd.), Pour un vocabulaire mystique au XVII siècle, Séminaire du Professeur Carlo Ossola, Torino, Aragno, 2005 , pp. 97-I 52.

23. Cfr. Renzo Rabboni, Laudari e canzonieri nella Firenze del '400. Scrittura privata e modelli nel Vat. Barb. Lat. 3679, Bologna, Clueb, 1991. Si veda, anche per ulteriori ragguagli bibliografici, Blake Wilson, Singing Poetry in Renaissance Florence. The Cantasi come Tradition (I375-I550), Firenze, Olschki, 2009.

24. In proposito si veda Paolo Cecchi, La fortuna musicale della "Canzone alla Vergine» petrarchesca e il primo madrigale spirituale, in Andrea Chegai e Cecilia Luzzi (a cura di), Petrarca in musica. Atti del Convegno Internazionale di Studi (Arezzo, I 8-24 marzo 2004), a cura di Lucca, LIM, 2005, pp. 247-292. Sul rilievo attribuito a Vergine bella nel contesto letterario cinquecentesco rinvio a Lorenzo Geri, $\mathrm{La}$ preghiera alla Vergine e il finale del libro nei commenti cinquecenteschi al Canzoniere, in Id. e Marco Grimaldi (a cura di), La lirica in Italia dalle origini al Rinascimento, Roma, Bulzoni, 2017, pp. I 27-145.

25. Sulle riscritture dei Salmi nel Cinquecento si veda Clara Leri, «La voce dello Spiro». Salmi in Italia tra Cinque e Settecento, Alessandria, Edizioni dell'Orso, 20 I I; Paolo Zaja, Salmi e lirica volgare nel Cinquecento, in Pietro Gibellini (dir.), La Bibbia nella letteratura italiana. V Dal Medioevo al Rinascimento, a cura di Grazia Melli e Marialuigia Sipione, Brescia, Morcelliana, 2013, pp. 549-568; Rosanna Morace, I Salmi tra Riforma e Controriforma, in Rosanna Alhaique Pettinelli, Rosanna Morace, Pietro Petteruti Pellegrino, Ugo Vignuzzi (a cura di), La Bibbia in poesia. Volgarizzamenti dei Salmi e poesia religiosa in età moderna, Roma, Bulzoni, 2015, pp. 55-8 I. Infine, si veda la tesi di dottorato di Ester Pietrobon, La penna interprete della cetra. I Salmi in volgare e la tradizione della poesia spirituale italiana nel Cinquecento, Università degli Studi di Padova, XXVII ciclo (Supervisore: Franco Tomasi).

26. Rime spirituali di sette poeti illustri, in Napoli, appresso Giovanni De Boy, I 599 (di questa rarissima stampa ho consultato l'esemplare della Biblioteca Nazionale di Napoli, 40.C.99, mutila delle cc. $*_{7-8}$ e I-6).

27. Sui Fiori ruscelliani si veda Quondam, Petrarchismo mediato, cit., pp. 195-199; Franco Tomasi, Distinguere $i$ «dotti da gl'indotti»: Ruscelli e le antologie di rime, in Paolo Marini e Paolo Procaccioli (a cura di), Girolamo Ruscelli. Dall'Accademia alla Corte alla Tipografia. Atti del Convegno internazionale di studi (Viterbo, 6-8 ottobre 20 I I), Manziana, Vecchiarelli, 20 I 2, vol. II, pp. 57 I-604, alle pp. 582-59I.

28. Rime di tre de' più illustri poeti dell' età nostra, cioè di Mons. Bembo, di Mons. della Casa et di Mons. Guidiccione, in Venetia, appresso Francesco Portonari, I 567.

29. Rime spirituali di sette poeti illustri, cit., c. ${ }^{*}{ }_{3} r-v$. Nella trascrizione di tutti i testi ho operato un moderato ammodernamento: distinzione tra $u$ e $v$; uniformazione 
grafica di $-i j$ in $-i i$; trasformazione del nesso $t i$ e $t t i+$ vocale in $z$, eliminazione dell' $h$ etimologica o paraetimologica; scioglimento delle abbreviazioni senza indicazioni; la nota tironiana sciolta in $e$ davanti a consonante, et davanti a vocale; conservazione delle grafie etimologiche, nonché di geminazioni e scempiature diverse dall'uso moderno; maiuscole e segni paragrafematici sono stati resi conformi all'uso attuale.

30. Sull'esperienza letteraria a Napoli nella seconda metà del Cinquecento si veda Ezio Raimondi, Il petrarchismo nell'Italia meridionale [1973], in Id. Rinascimento inquieto, Torino, Einaudi, I994, pp. 267-306; Amedeo Quondam, La parola nel labirinto. Società e scrittura del Manierismo a Napoli, Roma-Bari, Laterza, I 975 ; Giulio Ferroni-Amedeo Quondam, La «locuzione artificiosa». Teoria ed esperienza della lirica a Napoli nell'età del Manierismo, Roma, Bulzoni, I 973; Pasquale Sabbatino, Il modello bembiano a Napoli nel Cinquecento, Napoli, Ferraro, 1986. Per un affondo su alcuni temi riguardanti il contesto letterario napoletano del Cinqecento si vedano i saggi contenuti nei volumi di Tobia R. Toscano, Letterati corti accademie. La letteratura a Napoli nella prima metà del Cinquecento, Napoli, Loffredo, 2000 e L'enigma di Galeažo di Tarsia. Altri studi sulla letteratura a Napoli nel Cinquecento, Napoli, Loffredo, 2004.

3I. Indicativo quanto Ammirato scrisse nel dialogo Il Rota overo dell'imprese: «Sapete che oggimai la poesia è partita in due schiere; all'una diletta quello stile corrente e piano, che ha di quel del Petrarca, benché in lui tutte le cose concorsero. All'altra quel ritenuto e grave, strada accennata dal Bembo e poi con più studio seguita, anzi quasi di nuovo calcata dal Casa, in guisa con nuove foggie e maniere di dire andò da ciascun altro scostandosì (Scipione Ammirato, Il Rota overo dell'imprese, in Napoli, appresso Gio. Maria Scotto, I662, p. I44). Sul dialogo di Ammirato rinvio, anche per la bibliografia ivi citata, al saggio di Guido Arbizzoni, Imprese e poesia nel Rota di Scipione Ammirato, in Id., "Un nodo di parole e di cose». Storia e fortuna delle imprese, Roma, Salerno Editrice, 2002, pp. 37-57.

32. Questo l'ordine dei componimenti bembiani disposti nel volume: Già donna, hor dea, nel cui verginal chiostro; Signor, che parti et tempri gli elementi; Che gioverà da l'alma havere scosso; Signor, che per giovar sei Giove detto; Uscito fuor de la pregion trilustre; Signor del ciel s'alcun prego ti move; O sol, di cui questo bel sole è raggio; Se già ne l'età mia più verde et calda; Signor, quella pietà che ti constrinse. Per un inquadramento storico-critico dei singoli componimenti rimando all'edizione delle Rime bembiane curata da Andrea Donnini (Roma, Salerno Editrice, 2008).

33. Nella sezione dedicata a Guidiccioni risulta indicativa la ristampa dei tre sonetti in lode dell'Ochino, su cui cfr. supra.

34. Si legga quanto scrive Ammirato nei Ritratti, pubblicati postumi nel I 637 nel secondo tomo dei suoi Opuscoli, riguardo all'eccezionalità di Della Casa, il quale esplorò per primo nuovi sentieri poetici, discostantosi dall'imitazione pedissequa del modello petrarchesco: «Ciò che si pose a scrivere Giovanni Della Casa, nobile fiorentino, o versi e prose latine, o rime e prose toscane, o cose gravi o da scherzo, fece eccellentemente. E quel che è maraviglioso in lui fu che, avendo trovato tutti volti all'imitazione del Petrarca, solo egli fu primo ad uscir di questa via, trovando una maniera pellegrina, piena non meno di novità che di maestà, facendo le pose nel mezzo de' versi e tenendo sempre il lettore sospeso con piacere e con meraviglia» (Scipione Ammirato, Opuscoli [...] Tomo II, in Fiorenza, 
nella nuova Stamperia d'Amadore Massi e Lorenzo Landi, I637, p. 255 ). Sugli interventi di Ammirato intorno alla poesia lirica rinvio a Ferroni-Quondam, La «locu乏ৃione artificiosa», cit., pp. 73-91; Sabbatino, Il modello bembiano, cit., pp. 83-1ог.

35. Sull'importanza della silloge di Ammirato come testimone delle rime di Rota rimando alla Nota al testo di Berardino Rota, Rime, a cura di Luca Milite, Parma, Fondazione Pietro Bembo/Ugo Guanda Editore, 2000, pp. 667-669.

36. Basti qui ricordare il celebre caso dei rotiani Sonetti [...] in morte della S. Portia Capece sua moglie (Napoli, Cancer, i 560 ) curati e annotati da Ammirato, che allestì anche la prima edizione completa del canzoniere rotiano (Sonetti et canzoni, in Napoli, appresso Gio. Maria Scotto, I 560 ); cfr. ibid., pp. 644-645 e 675-680.

37. Cfr. la Nota sul testo in Vittoria Colonna, Rime, a cura di Alan Bullock, Roma-Bari, Laterza, I982, p. 28 5. Sulla sezione dedicata alla Colonna è intervenuta Concetta Cavallini, Le «pétrarquisme spirituel», Vittoria Colonna et les Rime spirituali di sette poeti illustri de Scipione Ammirato, in «Studi di letteratura francese», XXXIX (2014), pp. 9-25; Ead., Vittoria Colonna, femme/poète «spirituale» et le Rime spirituali di sette poeti illustri de Scipione Ammirato (I569), in Les Muses sacrées. Poésie et Théatre de la Réforme entre France et Italie, sous la direction de Rosanna Gorris Camos et Véronique Ferrer, Genève, Droz, 2016, pp. I 83-202.

38. Sulla fenomenologia poetica della gravitas rimando a Andrea Afribo, Teoria e prassi della gravitas nel Cinquecento, Firenze, Cesati, 200I. Sul dellacasismo tra Cinque e Seicento si veda dello stesso studioso Giovanni Della Casa tra Cinque e Seicento, nel suo Petrarca e petrarchismo. Capitoli di lingua, stile e metrica, Roma, Carocci, 2009, pp. 209-235. Sulla tradizione cinquecentesca della lirica morale, ai confini e in stretta relazione con quella religiosa, mi sia consentito rinviare a Pietro Giulio Riga, "Canterò di virtù l'alto valore». Appunti sulla tradiżione della lirica morale tra Cinque e Seicento, in La lirica in Italia, cit., pp. 2 I I-235.

39. Vitale sarà poi noto per aver ingaggiato agli inizi del Seicento una polemica con Giovan Battista Marino, i cui contorni restano ancora nell'ombra; un'analisi dei testi in Francesco Tateo, Giambattista Vitale da Foggia e le polemiche marinistiche, in «Lingua e storia in Puglia», I (1974), pp. 39-54.

40. Rime spirituali di diversi eccellenti poeti toscani raccolte da M. Giovanbattista Vitale, in Napoli, appresso Horatio Salviani, I 574, c. A2r-v.

4I. Per un inquadramento storico-critico della lirica dolciana, soprattutto per i sonetti di materia spirituale editi nella silloge giolitina del I 547 , rimando al documentato studio di Paolo Marini, «Non per buman lavoro». Note su Dolce lirico e la questione dei sonetti spirituali, in Per Lodovico Dolce. Miscellanea di studi I. Passioni e competenze del letterato, a cura di Id. e Paolo Procaccioli, Manziana, Vecchiarelli, 20I6, pp. 4I-89. Inoltre, in vista dell'edizione della lirica dolciana si veda dello stesso studioso, Per l'edizione commentata della lirica di Lodovico Dolce, in Béatrice Alfonzetti, Guido Baldassarri e Franco Tomasi (a cura di), I cantieri dell'italianistica. Ricerca, didattica e organizzazione agli inizi del XXI secolo, Atti del XVII congresso dell'ADI (Roma Sapienza, i 8-2 I settembre 2013), Roma, Adi editore, 2014, pp. I-8 (pubblicazione elettronica in formato pdf disponibile sul sito web di libero accesso http://www.italianisti.it/). 
42. Si veda l'edizione moderna: Salmi penitenziali di diversi eccellenti autori, edizione critica a cura di Rosanna Morace, Pisa, ETS, 2017, in particolare l'Introduzione alle pp. VII-XLIV. Sui testimoni a stampa della raccolta rinvio alla Nota al testo, ibid., pp. XLV-LXIII. Contestualmente si rimanda anche al saggio di Paolo Zaja, Francesco Turchi e $i$ Salmi penitentiali di diversi eccellenti autori (Venezia, Is68), «Quaderni veneti», 3 (2014), pp. $65-73$.

43. Su questi aspetti mi limito a rinviare a Gigliola Fragnito, La Bibbia al rogo. La censura ecclesiastica e i volgarizzamenti della Scrittura (I47I-1605), Bologna, Il Mulino, I 997 e Vittorio Frajese, Nascita dell'Indice. La censura ecclesiastica dal Rinascimento alla Controriforma, Brescia, Morcelliana, 2006.

44. Cfr. le indicazioni contenute nel volume Giovanni Filoramo (dir.), Storia della direzione spirituale. III L'età moderna, Gabriella Zarri (a cura di), Brescia, Morcelliana, 2008.

45. Sulla straordinaria fase di crescita del libro spirituale e sul ruolo dell'editoria specializzata nella pubblicazione di opere di carattere religioso si veda Danilo Zardin, Mercato librario e letture devote nella svolta del Cinquecento tridentino. Note in margine ad un inventario milanese di libri di monache, in Nicola Raponi e Angelo Turchini (a cura di), Stampa, libri e letture a Milano nell'età di Carlo Borromeo, Milano, Vita e Pensiero, I992, pp. I 35-246; Ugo Rozzo, Linee per una storia dell'editoria religiosa in Italia (1465-1600), Udine, Arti Grafiche Friulane, I993; Ugo Rozzo e Rudj Gorian (a cura di), Il libro religioso, id. Milano, Edizioni Sylvestre Bonnard, 2002; Edoardo Barbieri, Fra tradizione e cambiamento: note sul libro spirituale del XVI secolo, in Edoardo Barbieri e Danilo Zardin (a cura di), Libri, biblioteche e cultura nell'Italia del Cinque e del Seicento, Milano, Vita e Pensiero, 2002, pp. 3-62; Danilo Zardin, Nutrire con frutto l'esperienza. Il libro devoto nell'Italia del Cinquecento, in Aspirazioni e devozioni: Brescia nel Cinquecento tra pregbiera e eresia, a cura di Ennio Ferraglio, Milano, Electa, 2006, pp. 36-5 I; Gabriella Zarri, Libri di spirito. Editoria religiosa in volgare nei secoli $X V$-XVII, Torino, Rosenberg \& Sellier, 2009, in particolare pp. $103-\mathrm{I} 64$.

46. Sul Giolito editore di testi sacri si veda, anche per ulteriori rimandi bibliografici, Quondam, Note sulla tradizione, cit., pp. 162-163.

47. Questa come la citazione precedente è tratta dai Salmi penitentiali di diversi eccellenti autori. Con alcune rime spirituali di diversi Illust. Cardinali, di Reverendissimi Vescovi et d'altre persone ecclesiastiche; scelti dal Reverendo P. Francesco da Trivigi Carmelitano. Nuovamente da lui corretti et ristampati, in Vinegia, appresso Gabriel Giolito de' Ferrari, I 572, cc. *iiij $r$ - vj $r$.

48. Ossia le Stanze in lode della castità in risposta di quelle dell'Illustriss. Cardinal Bembo.

49. Si tratta di una versione scorciata, di 42 ottave, delle Lagrime di San Pietro, che Turchi attribuisce correttamente, nell'edizione del 1572, a Lugi Tansillo: Le lagrime di San Pietro, secondo alcuni del Reverendiss. Cardinale de' Pucci, ma secondo la verità del S. Luigi Tansillo. Sulle vicende redazionali dell'opera $c f r$. Tobia R. Toscano, Note sulla composizione e la pubblicazione de Le lagrime di San Pietro di Luigi Tansillo (con inediti), in Rinascimento meridionale e altri studi, raccolta di studi pubblicata in onore di Mario Santoro, Napoli, Società Editrice Napoletana, I987, pp. 437-46r. Da 
ultimo si veda l'edizione offerta da Rosanna Morace, La prima redazione delle Lagrime di San Pietro di Luigi Tansillo con le varianti dei principali testimoni, in appendice ai Salmi penitenziali, cit.

50. Cfr. Giovanni Della Casa, Rime, a cura di Stefano Carrai, Torino, Einaudi, 2003, pp. 2 I 2-2 I 4 (la citazione a p. 2 I 2). Sulla scelta e sulla provenienza dei testi riuniti nella raccolta rinvio a Rosanna Morace, Introduzione e Nota al testo, in Salmi penitenz̧iali, cit., pp. XXXVIII-XLIV e LIV-LVIII.

5. I. Dalla nota A' Signori lettori edita in Le Muse sacre. Scelta di rime spirituali de' più eccellenti autori d'Italia, del Signor Pietro Petracci, in Venetia, appresso Evangelista Deuchino e Giovan Battista Pulciano, i608, cc. a7r-v.

52. Ibid. Sui temi presenti nella raccolta è intervenuto Salvatore Ussia, Le Muse sacre. Poesia religiosa dei secoli XVI e XVII, con schede di Elenora Bellini, antiche riproduzioni dalla Biblioteca Molli, Borgomanero, Fondazione Achille Marazza, I999.

53. Degne di nota sono le lettere di Grillo a Petracci, che concernono l'iter editoriale del volume delle Lettere (I6 I 2) e dei Pietosi affetti, nonché lo scambio di rime (cfr. Angelo Grillo, Lettere, in Venetia, appresso Bernardo Giunti, Gio. Battista Ciotti e compagni, I6o8 e Id., Delle lettere [...] volume secondo, in Venetia, per Evangelista Deuchino, 16 г 2, ad indicem).

54. Mentre approntava il volume delle Muse sacre, Petracci allestiva per conto di Ciotti un'altra importante antologia poetica, che mi propongo di esaminare in altra sede, nella quale venivano raccolti i più noti madrigalisti di inizio secolo: Ghirlanda dell'aurora, scelta di madrigali de' più famosi autori di questo secolo (I (609).

55. Sui madrigali di Grillo si veda Giulia Raboni, Il madrigalista genovese Livio Celiano e il benedettino Angelo Grillo, «Studi secenteschi», XXXII (I99I), pp. I 37I88. Per un più ampio inquadramento della poesia del benedettino ligure rimando alle prospettive di ricerca offerte da Francesco Ferretti, Le Muse del Calvario. Angelo Grillo e la poesia dei benedettini cassinesi, Bologna, Il Mulino, 20 I 2.

56. Per l'attività e per il catalogo delle edizioni dello stampatore veneziano attivo tra la fine del Cinquecento e i primi decenni del Seicento si rinvia a Dennis E. Rhodes, Giovan Battista Ciotti (1562-1627?): Publisher extraordinary at Venice, Venezia, Marcianum Press, 2013.

57. Cfr. Lorenzo Sacchini, Sulle Rime del perugino Filippo Alberti (I548-16I2), «Aevum», 88 (2014), 3, pp. 637-663.

58. Rime di elevati ingegni de la città di Udine raccolte da Giacomo Bratteolo, in Udine, appresso Giovan Battista Natolini, I 597.

59. Gabriele Finmma, Rime spirituali [...] novamente impresse con gli argomenti di Pietro Petracci, in Venetia, presso Giovan Battista Ciotti, I606, c. A2v (si tratta del secondo volume di un interessante progetto editoriale in sei tomi che comprendeva sei autori di poesia religiosa: Scielta di varie poesie sacre di diversi eccellenti autori in lode di N. Signor et di Maria Vergine e d'altri santi. Gabriello Chiabrera. Gabriello Fiamma. 
Pietro Giulio Riga

Giovanni Ralli. Bartolomeo Barco. Cornelio Tirabosco. Nicolo Negri, in Venetia, presso Bernardo Giunti, Giovan Battista Ciotti e compagni, I604-I608). Questa edizione delle Rime spirituali veniva privata del paratesto autoesegetico per ragioni illustrate dall'editore nella prefazione $A$ chi leggerà: «E se bene furono poste in luce con la sposizione dell'istesso piena d'alta dottrina, nulladimeno, perciò che il volume era discommodo in quella primiera forma, ho voluto appresentarvelo in questa ch'ora si vede, avendo tralasciata la dichiarazione solo, acciò che non crescesse il libro con disparutezza, essendo però aggiunti gli argomenti in fronte a ciascuna composizione cavati da gentile Spirito, secondo l'uso moderno, li quali non deveranno parer lunghetti poiché a simil genere di componimenti par che sia più necessario dilatarsi un poco che ne' suggetti amorosi» (ibid. cc. A $5 v$-A6r).

6o. Sulla lirica sacra mariniana e sulla sua diffusione nelle raccolte collettive anteriori al I6I4, anno di uscita della terza parte della Lira, si veda Alessandro Martini, Le Divozioni del Marino, in Matteo M. Pedroni (a cura di), «Parlar l'idioma soave». Studi di filologia, letteratura e storia della lingua offerti a Gianni A. Papini, Novara, Interlinea, 2003, pp. I 8 I-I 95 .

61. Questo il titolo completo delle due parti: Nuovo concerto di rime sacre, tutte ripiene di bellissimi et esquisiti concetti composte da' più eccellenti poeti d'Italia. Sopra i principali misteri della vita e morte di Christo Nostro Signore e della Regina de' Cieli, in Venetia, appresso Antonio Pinelli, r6r6; Nuovo concerto di rime sacre composte in lode de' santi, et in varii soggetti spirituali e morali, da' più eccellenti poeti d'Italia, cosi antichi, come moderni. Parte seconda, in Venetia, appresso Antonio Pinelli, I6I6 (ho consultato l'esemplare della Biblioteca Apostolica Vaticana, Stamp. Barb. JJJ.I. I 7).

62. Nuovo concerto, cit., c. †3r. La dedicatoria della seconda parte è indirizzata a Marco Priuli e porta la stessa data della prima (24 marzo i6 I6).

63. Antonio Bruni, Selva di Parnaso [...] con brevi argomenti del Cavalier Aurelio Alconi, in Venezia, appresso i Dei, i6 is. Su Bruni, anche per la bibliografia ivi citata, rimando al saggio di Lorenzo Geri, Le Epistole eroiche di Antonio Bruni tra Umoristi e Caliginosi, in Clizia Gurreri e Ilaria Bianchi (a cura di), Le virtuose adunanze. La cultura accademica tra XVI e XVIII secolo, Prefazione di Giulio Ferroni. Introduzione di Gian Mario Anselmi, Avellino, Edizioni Sinestesie, 2015, pp. $173-193$.

64. Rosario dalle stampe de tutti poeti e poetesse, antichi e moderni, cinquecento di numero. Tomo ottavo e quadro del giardino di tutte le scientie di fra Mauritio di Gregorio, Napoli, Carlino, I6ı4, c. aı $v$ ('opera è rarissima: ho individuato due soli esemplari, entrambi conservati nella Biblioteca Nazionale di Napoli con segnatura B. Branc. 79.A.36 e 79.A.38). In coda al Rosario si trova un Sogno di F. Mauritio Academico Ocioso al Rosario di soo poeti per ordine delle muse che, sulla spinta di una tensione enciclopedica al catalogo di autori e testi antichi, ripercorre la disputa sorta nell'Accademia napoletana degli Oziosi sul primato tra poesia e oratoria; cfr. Daniela Caracciolo, Il «Sogno» di un collezionista del Seicento napoletano. Maurizio Di Gregorio tra riscrittura e plagio, "Parole rubate. Rivista internazionale di studi sulla citazione», 4 (20I I), pp. I 3 I-I 47. Sulla figura di Di Gregorio, attivo tanto sul versante del collezionismo enciclopedico quanto su quello della retorica sacra, rimando a Maurizio Di Gregorio, "Le coselline di un ometto curioso». L'Idea per fare 
le gallerie universali di tutte le cose del mondo, naturali, artificiali e miste, a cura di Daniela Caracciolo, Nota filologica di Girolamo De Miranda, Taranto, Congedo, 2008; Erminia Ardissino, Il condottiere dei predicatori. Maurizio Di Gregorio e la retorica sacra di primo Seicento, in Paola Baioni (a cura di), I domenicani e la letteratura, introduzione di Carlo Delcorno, Roma-Pisa, Serra, 2016, pp. 69-80.

65. Rosario dalle stampe, cit., c. a3r.

66. Ibid., c. a2r-v.

67. Sulla tipologia poetica del rosario si veda Quondam, Note sulla tradizione, cit., pp. 207-208. Sul rilancio a fine Cinquecento della devozione rosariana rimando ai saggi contenuti nel volume Riccardo Barile (a cura di), Il Rosario tra devozione e riflessione. Teologia, storia, spiritualità, numero monografico di «Sacra Doctrina», LIV (2009), pp. 275-297 (in particolare, sul versante letterario, i contributi di Erminia Ardissino, Il rosario nella predicazione tra Cinque e Seicento, ibid., pp. 275-296; Mario Rosa, I trionfi del Rosario nella letteratura religiosa italiana della Controriforma, ibid., pp. 298-3I3).

68. Nuova raccolta di lagrime di più poeti illustri, in Bergamo, per Comin Ventura, I 593. Sul genere poetico delle lacrime si veda Quondam, Note sulla tradizione, cit., pp. I92-196 e Angelo Alberto Piatti, «E l'uom pietà da Dio, piangendo impari». Lacrime e pianto nelle rime sacre dell'età del Tasso, in Rime sacre tra Cinquecento e Seicento, cit., pp. 53-106.

69. Su questa antologia, come sulle altre curate da Silvestro da Poppi nel primo decennio del secolo, si veda Armando Maggi, Francesco d'Assisi e le stimmate alla luce del Barocco. Sette canzoni di sette famosi autori (I606) e Rime spirituali di diversi autori (I606) raccolte da F. Silvestro da Poppi minore osservante, in «Studi secenteschi», XLIX (2008), pp. 79-I 30. Sulla letteratura del Seicento dedicata a San Francesco si veda inoltre Maria Luisa Doglio, Immagini di San Francesco nella letteratura del Seicento, in «Rivista di Storia e Letteratura Religiosa», XXV (1989), pp. 423-443; Giorgio Forni, Florilegi fiorentini del primo Seicento in lode di San Francesco, in Rime sacre tra Cinquecento e Seicento, cit., pp. I4I-I 85 .

70. Una panoramica sul fenomeno nel saggio di Maiko Favaro, «Erger sui tempii di vivace fede». Sulla declinazione sacra del «tempio di rime» fra ', 00 e '600, «Rassegna europea della letteratura italiana», 44 (2014), pp. 45-58. In generale, sulla tipologia del tempio di rime rimando a Monica Bianco, Il «Tempio» in onore: parabola di un genere antologico cinquecentesco, in Donatella Rasi (a cura di), Miscellanea di studi in onore di Giovanni Da Pozzo, Roma-Padova, Antenore, 2004, Pp. I63-189.

71. Del Tempio armonico della beatissima Vergine (Roma, Niccolò Muzi, I 599) si dispone di un'edizione moderna: Elisabetta Crema, Il Tempio armonico di Giovenale Ancina: edizione e commento, tesi di dottorato di ricerca. Tutor: C. Berra, coordinatore: F. Brioschi, Milano, Università degli Studi di Milano, 2005 (da cui si citerà). Sull'opera dell'Ancina si veda sia sul fronte musicale che su quello storicoletterario: Arnaldo Morelli, Il Tempio armonico. Musica nell'Oratorio dei Filippini in Roma (I575-1705), «Analecta musicologica», XXVII (I 99I), pp. I-2 I6; Maria Luisa Doglio, Il Tempio armonico di Giovenale Ancina. Dal Petrarca «travestito» alla lauda 


\section{Pietro Giulio Riga}

spirituale alla «canzonetta ariosa», nel suo «Scrivere di sacro». Forme di letteratura religiosa dal Duecento al Settecento, Roma, Edizioni di Storia e Letteratura, 20I4, pp. 3 I-4I; Elisabetta Crema, Aspetti di petrarchismo sacro: il Tempio armonico di Giovenale Ancina, "Ambra», V (2005), pp. 20-39. Ead., Pubblicare testi musicali: il Tempio armonico di Giovenale Ancina, «Moderna», X (2008), pp. I I7-I 26. Inoltre, si vedano i saggi contenuti in Carla Bianco (a cura di), Il «Tempio armonico». Giovenale Ancina e le musiche devozionali nel contesto internazionale. Convegno internazionale di studi, Saluzzo 8-1o ottobre 2004, Lucca, Libreria musicale italiana, 2006. Inoltre, ai fini di una più corretta contestualizzazione storico-culturale del Tempio armonico si veda Anne Piéjus, Musique et dévotion à Rome à la fin de la Renaissance. Les laudes de l'Oratoire, Turnhout, Brepols, 2014.

72. Crema, Il Tempio armonico, cit., p. 320.

73. Cito dal Discorso apologetico per difesa dell'opra dagli impetuosi, agri e calunniosi impugnatori (CRema, Il Tempio armonico, cit., p. 35 I).

74. Su questa raccolta rinvio a Monica Bianco, Il «Tempio» a Geronima Colonna D'Aragona ovvero la conferma di un archetipo, in «I più vaghi e i più soavi fiori», cit., pp. I47-I 8 I.

75. Crema, Il Tempio armonico, cit., pp. 291-293.

76. Il Sacro Tempio dell'Imperatrice de' Cieli Maria Vergine santissima. Fabricato de' più purgati carmi c'babbiano composti i primi poeti d'Italia, cosi antichi come moderni. Fatica del Confuso Academico Ordito [Carlo Fiamma], in Vicenza, appresso Francesco Grossi, I6 I 3. La raccolta, curata dal nipote di Gabriele Fiamma, ospita 55 autori per un totale di 28 I testi. Conta dare nota della logica strutturale dell'antologia, frutto di una precisa suddivisione tematica dei testi, segnalata in apertura da una Tavola de' capi sopra quali è scritta la presente opera, che comprende nuclei come l'Invocatione, la Visitatione, lo Stabat mater e le Lacrime di Maria Vergine fino alle cronache locali come la Historia della B. Vergine del Monte di Berga di Vicenza. Nella silloge viene attribuito un rilievo speciale a poeti piuttosto noti negli ambienti religiosi e attestati, lo si è detto, nelle antologie spirituali di area veneta: Crisostomo Talenti (24), Maurizio Moro (40) e lo stesso Carlo Fiamma (30). Petrarca compare solo con Vergine bella mentre risultano del tutto assenti «campioni» del passato come Malipiero e Colonna. 